\title{
On the Control of a Queueing System with Aging State Information
}

\author{
Martijn Onderwater ${ }^{* 1,2}$, Sandjai Bhulai ${ }^{\dagger 2}$, and Rob van der $\mathrm{Mei}^{\ddagger 1,2}$ \\ ${ }^{1}$ Center for Mathematics and Computer Science (CWI) \\ Science Park 123, 1098 XG Amsterdam \\ The Netherlands \\ ${ }^{2}$ VU University Amsterdam, Faculty of Sciences \\ De Boelelaan 1081a, 1081 HV Amsterdam \\ The Netherlands
}

June 2, 2015

\begin{abstract}
We investigate control of a queueing system in which a component of the state space is subject to aging. The controller can choose to forward incoming queries to the system (where it needs time for processing), or respond with a previously generated response (incurring a penalty for not providing a fresh value). Hence, the controller faces a trade-off between data freshness and response times. We model the system as a complex Markov Decision Process, simplify it, and construct a control policy. This policy shows near-optimal performance and achieves lower costs than both a myopic policy and a threshold policy.
\end{abstract}

Keywords: Markov Decision Processes, Controlled Queueing System, Aging State Information, One-step Policy Improvement, Ordered Performance Curve, Value Iteration, Difference Equations, Optimization

\section{Introduction}

In this paper we study the control of a queueing system in which part of the data is subject to aging. The system contains a controller that must provide responses to incoming queries, either using aged data or with a newly generated value from the queueing system. Using the queueing system ensures a fresh response to the query, whereas generating the response takes some time, particularly if the load on the system is high. Alternatively, the controller may use a previously generated value, which is, however, not as fresh as a response from the queueing system. Consequently, the controller faces a trade-off between data freshness and query response times.

\footnotetext{
*m.onderwater@cwi.nl

${ }^{\dagger}$ s.bhulai@vu.nl

${ }^{\ddagger}$ r.d.van.der.mei@cwi.nl
} 


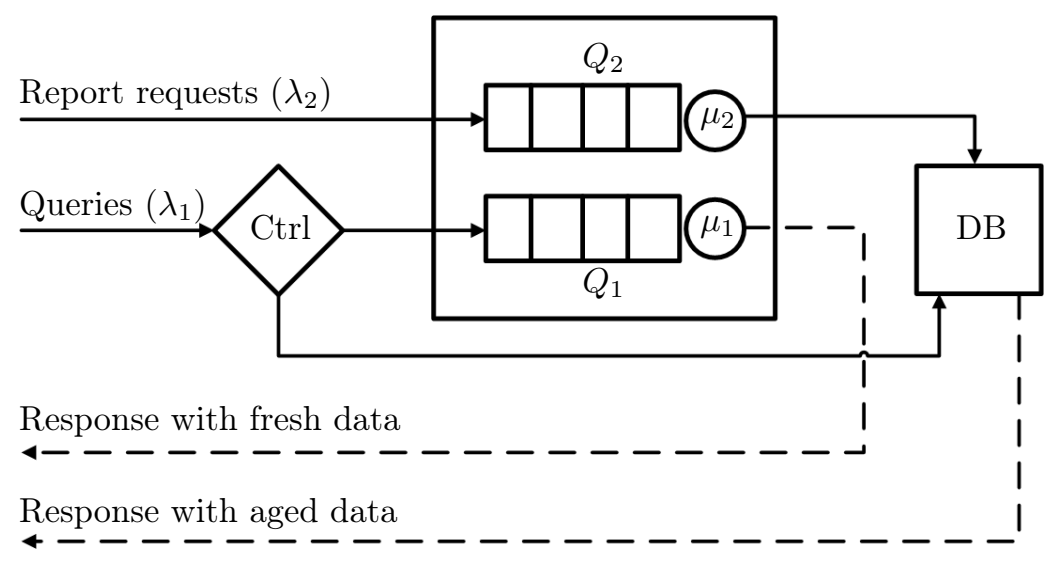

Figure 1. The controller (Ctrl) assigns incoming queries to either $Q_{1}$ in the queueing system, or to the DB. In the first case, the query gets a fresh response, but has to wait some time before it is generated. In the second situation, the systems returns a previously generated (and thus aged) response immediately. The DB is regularly refreshed with fresh values (reports) from $Q_{2}$

We illustrate the system in Fig. 1, where a controller Ctrl handles incoming queries that require a response. The controller uses a policy to determine whether a query receives a response with fresh data, or with aged data. In the first case, the query is forwarded to a queue $Q_{1}$ where the query is eventually serviced. In the second case, the query is immediately answered with a known, aged, response that is stored in, e.g., a database (DB). The DB is regularly refreshed by reports from a queue $Q_{2}$. For modelling purposes we assume that both queries and report requests arrive according to a homogeneous Poisson Process with rate $\lambda_{1}$ and $\lambda_{2}$, respectively. Also, we assume that the processing time in the queues is exponentially distributed with parameter $\mu_{1}$ (for queries) and $\mu_{2}$ (for report requests).

An illustration of the interaction between queries, reports, and the age of the latest value in the DB is shown in Fig. 2. At time 1 a job is completed at the server of $Q_{2}$ (resulting in a report) and sets the age to 0 . This age then increases linearly until the next report is generated at time 4 . Meanwhile, at time 1.5 a query arrives at the controller, at which moment the age of the latest value in the database is 0.5 . Then at time 3 the second query arrives, which sees the most recent value in the database at age 2. Query 3 arrives after the second report is generated, at which point the value in the database has age 1 . Report 3 at time 6 refreshes the database again and sets the age to 0 . Note that the graph does not show which decisions the controller takes on arrival of a query.

The choice between using instantly available aged values and generating fresh ones regularly occurs in practice. For instance, obtaining fresh measurements from a wireless sensor network is relatively time-consuming due to the wireless transmissions across the network. Therefore, a gateway to the sensor network can retain previously generated values for answering queries, and thus faces a tradeoff similar to the one we consider in this paper. A second example is a web server responsible for retrieving a web page. It either instantly obtains the requested page from a local cache, or takes some time to regenerate a fresh version of the page. Again, the choice of the web server is based on a trade-off similar to the description above.

Addressing the trade-off is traditionally done using a threshold policy, see for instance [1] and [16] in the context of the web server example. Namely, when the age of the database value exceeds a threshold, retrieval of fresh data is initiated, and otherwise the cached value is used. Although such systems are commonly used, there is room for improvement by setting a dynamic threshold 


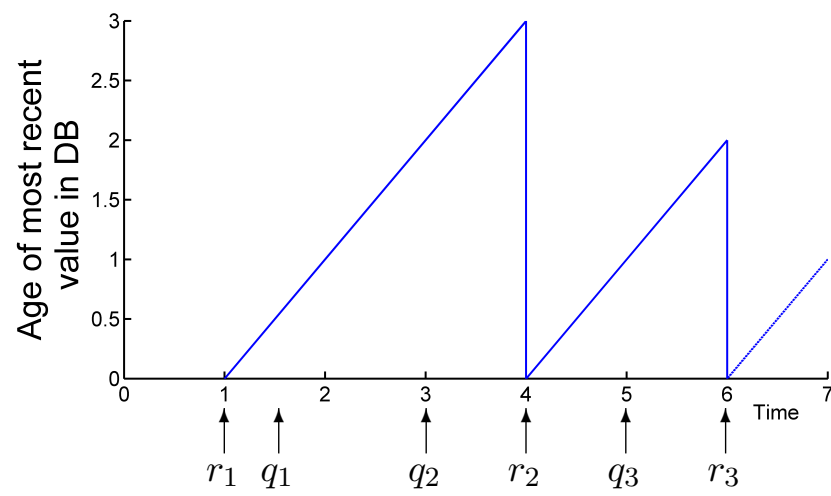

Figure 2. Interaction between queries $\left(q_{1}, \ldots, q_{3}\right)$, reports $\left(r_{1}, \ldots, r_{3}\right)$, and the age of the latest value in the database. In the graph, three reports arrive (at times 1, 4, and 6) that reset the age to 0 . In between reports, the age increases linearly with time. Upon a query arrival, the controller sees the latest value in the database at a certain age and uses that age to take its decisions. For instance, query $q_{2}$ arrives at time 3 , at which moment the most recent value in the database has age 2

based on the expected response time in the system of the query. In cases where the information retrieval is time-consuming, using a database value that is slightly above the threshold value might be acceptable. Hence, there is a trade-off between using a database value that has an age that is (slightly) above the threshold value and the expected response time of query when it is handled by the system.

In this paper, we formulate the scenario above as a three-dimensional Markov Decision Process (MDP). The refresh of the database causes subtle interactions between the state variables, making the problem hard to solve analytically. Therefore, we construct an approximate model that captures these dynamics in a simpler way, allowing for an analytical solution. The analysis of the simpler model relies on differencing techniques to deal with several inhomogeneous terms. After finding the analytical solution, we apply one-step policy improvement to obtain an improved policy. Finally, we numerically compare this policy to the optimal policy, as well as to a myopic policy and to a traditional age-threshold policy. The improved policy achieves near-optimal performance, and performs better than the myopic and the age-threshold policy.

The scenario described above is characterized by three distinctive components: (1) a queueing system, (2) a database that is periodically refreshed from the queueing system, and (3) the controller assigning queries to either of the two other components. Despite a thorough literature review, we did not find any research with the same combination of components (apart from [12], where we investigate the same scenario using a different model). The caching application mentioned before is related, but seems to be not used together with a queueing system. From a queueing theoretic approach, the papers by [7] and [13] are somewhat similar to our situation. They deal with several servers for which aged information about the loads is available and, as in our approach, this aged information is periodically updated by the queues via reports. Their system, however, does not contain a database, but has multiple queues that can serve the incoming jobs. Therefore, the controller decides which of the queues to use based on the aged load information, and thus addresses a problem different from ours.

Our approach relies on the one-step policy improvement technique, introduced by [14]. As a starting point we use the so-called Bernoulli policy, because it decouples the queueing system in Fig. 1 from the DB and allows for an explicit analysis. This decoupling aspect has been used in, for instance, [15] where the authors derive state-dependent routing schemes for high-dimensional circuit-switched telephone networks, relying on the Bernoulli policy to allow an analysis of individual communication 
lines. Other applications include the control of traffic lights [8], inventory control [21], routing of telephone calls in call centers [5], and controlled queueing models [3, 17].

Our contributions in this paper are the following: first, we introduce a control problem of a queueing system in which part of the state space is subject to aging. Then, from a methodological point of view, we provide a clever strategy for reducing the dimensionality of a MDP. Furthermore, during our derivation of a control policy, we present an intuitive and computationally efficient method to determine one of the parameters. Finally, we show that combining the latter two methodological approaches yields a near-optimal control policy for our queueing system.

The remainder of the paper is structured as follows. Section 2 introduces the MDP used to model the scenario above, and Section 3 illustrates the three steps of our approach to finding a near-optimal control policy. Then, Section 4 presents the first of these steps, detailing how the approximate model is constructed. The second step, finding a solution to the approximate model, is in Section 5 . Section 6 contains the third and final step, describing the derivation of our near-optimal control policy. Numerical experiments with this policy are presented in Section 7, as well as a closer look at the optimal policy. We finish with conclusions and future research directions in Section 8.

\section{Model formulation}

The trade-off we discuss in this paper is between data freshness and query response times. Here, we assume that the query response time is proportional to the current workload of the system, i.e., the number of queries plus the number of report requests in the system. The decision (using the $\mathrm{DB}$, or the queueing system) thus depends on the number of queries in the system, on the number of report requests, and on the age of the most recent value in the DB. In order to analyze decision policies, we formulate the scenario as a Markov Decision Process (MDP). As state space we use $\mathcal{X}=\mathbb{N}_{0} \times \mathbb{N}_{0} \times \mathbb{N}_{0}$, where $(i, j, N) \in \mathcal{X}$ denotes a system containing $i$ queries and $j$ report requests, and where the latest report refreshed the DB $N$ time units ago. The controller can choose actions $a$ from $\mathcal{A}=\left\{Q_{1}, \mathrm{DB}\right\}$, where $Q_{1}$ indicates forwarding of the query to $Q_{1}$ (see Fig. 1 ). The cost function $c(i, j, N ; a)$ incorporates the costs of each action available to the controller:

$$
c(i, j, N ; a)= \begin{cases}\gamma_{1}(i+1)+\gamma_{2} j+\gamma_{3}, & \text { if } a=Q_{1}, \\ (N-T)^{+}, & \text {if } a=\mathrm{DB} .\end{cases}
$$

Here, $\gamma_{1}(i+1)+\gamma_{2} j+\gamma_{3}$ is a weighted sum (with weights $\gamma_{1}, \gamma_{2}, \gamma_{3} \in \mathbb{R}$ ) of the number of queries and report requests in the system, reflecting the workload of the system after assigning a new query to it. The term $(N-T)^{+}$in the cost function is a penalty for returning a stale value from the DB instead of a fresh value. The parameter $T$ indicates a threshold below which the latest value in the DB is recent enough to answer the query. Note that we took $\gamma_{1}(i+1)$ rather than $\gamma_{1} i$ in the cost function, because we include the query that is about to be assigned to $Q 1$ when that action is chosen. Additionally, the resulting expression for the improved policy closely resembles a simple myopic policy that we investigate numerically, thereby further emphasizing the potential of the improved policy.

The state space, the action set, the transition rates, and the cost function define the Markov Decision Process. More explicitly, the optimality equation of the MDP can be formulated as follows:

$$
\begin{aligned}
g+V(i, j, N) & =\lambda_{2} V(i, j+1, N+1)+\mu_{1} V(i-1, j, N+1) \mathbb{1}_{\{i>0\}}+\mu_{2} V(i, j-1,0) \mathbb{1}_{\{j>0\}} \\
& +\left(1-\lambda_{1}-\lambda_{2}-\mu_{1} \mathbb{1}_{\{i>0\}}-\mu_{2} \mathbb{1}_{\{j>0\}}\right) V(i, j, N+1) \\
& +\lambda_{1} \min \left\{\gamma_{1}(i+1)+\gamma_{2} j+\gamma_{3}+V(i+1, j, N+1) ;(N-T)^{+}+V(i, j, N+1)\right\},
\end{aligned}
$$


with $V(i, j, N)$ the relative value function and $g$ the time-average costs. The uniformization term (see $[11,18])$ is formed by $\left(1-\lambda_{1}-\lambda_{2}-\mu_{1} \mathbb{1}_{\{i>0\}}-\mu_{2} \mathbb{1}_{\{j>0\}}\right) V(i, j, N+1)$, assuming that parameters $\lambda_{1}, \lambda_{2}, \mu_{1}, \mu_{2}$ are normalized such that $\lambda_{1}+\lambda_{2}+\mu_{1}+\mu_{2}=1$. Hence, we can regard these parameters as transition probabilities and Eq. (2) as a discrete-time model. Also note that $N$ measures the number of uniformized time steps since the generation of the last report, and not "real" time. Finally, we assume the stability conditions $\rho_{1}:=\lambda_{1} / \mu_{1}<1$ and $\rho_{2}:=\lambda_{2} / \mu_{2}<1$ hold.

\section{Obtaining the near-optimal policy}

Ideally, we would like to solve the optimality equation (2) analytically and obtain an expression for the relative value function (and, consequently, for the optimal policy). However, the optimality equation has two complicating aspects that prevent us from doing so. First, it contains the decision capturing the trade-off faced by the controller, which involves evaluation of a minimization term. Moreover, the inhomogeneous terms $\gamma_{1}(i+1)+\gamma_{2} j+\gamma_{3}$ and $(N-T)^{+}$in this minimum add to the complexity of the model. Second, the state space variables interact with each other, mainly through points depending on their neighbors (i.e., in Eq. (2), $V(i, j, N)$ depends on neighbors $V(i, j+1, N+$ 1), $V(i-1, j, N+1), V(i, j, N+1)$, and $V(i+1, j, N+1))$. An exception to this is the term $\mu_{2} V(i, j-1,0)$, which causes a complex relation between $j$ and $N$.

$$
V(i, j, N) \stackrel{I}{\longrightarrow} \tilde{V}(i, j) \stackrel{I I}{\longrightarrow} \tilde{V}^{\alpha}(i, j) \stackrel{I I I}{\longrightarrow} \pi^{\prime}
$$

Figure 3. Notation used in the steps $(I)-(I I I)$ of finding a near-optimal policy $\pi^{\prime}$ for Eq. (2)

In this paper we derive an approximate model to the original problem, ultimately resulting in a nearoptimal control policy. In the coming sections we take the following steps (illustrated in Fig. 3):

I We start in Section 4 with a modification of the optimality equation (2), obtained by removing the $N$-dimension, resulting in an MDP for an approximation to $V(i, j, N)$ (denoted by $\tilde{V}(i, j)$ ).

II In Section 5 we choose a policy for this new MDP and solve it analytically, yielding a solution $\tilde{V}^{\alpha}(i, j)$. Here, $\alpha$ is the parameter of a Bernoulli routing policy.

III Finally, in Section 6, we apply one-step policy improvement by inspecting the minimum in Eq. (2), substituting $\tilde{V}^{\alpha}(i, j)$ for $V(i, j, N)$. This results in an improved policy, denoted by $\pi^{\prime}$.

\section{Model approximation (Step I)}

Looking at Eq. (2), we see that $N$ is in the state space to accommodate the penalty term $(N-T)^{+}$. Therefore, if we replace the $(N-T)^{+}$by a suitable constant $C$, the $N$ can be removed from the state space. Introducing the constant $C$ in Eq. (2) yields

$$
\begin{aligned}
\tilde{g}+\tilde{V}(i, j) & =\lambda_{2} \tilde{V}(i, j+1) \\
& +\mu_{1} \tilde{V}(i-1, j) \mathbb{1}_{\{i>0\}}+\mu_{2} \tilde{V}(i, j-1) \mathbb{1}_{\{j>0\}} \\
& +\left(1-\lambda_{1}-\lambda_{2}-\mu_{1} \mathbb{1}_{\{i>0\}}-\mu_{2} \mathbb{1}_{\{j>0\}}\right) \tilde{V}(i, j) \\
& +\lambda_{1} \min \left\{\gamma_{1}(i+1)+\gamma_{2} j+\gamma_{3}+\tilde{V}(i+1, j) ; C+\tilde{V}(i, j)\right\} .
\end{aligned}
$$


As it turns out, the constant $C$ does not affect our near-optimal policy, so assigning a value to it is not strictly necessary (in Section 6.1 the term $(N-T)^{+}$is reintroduced). However, the idea of reducing the state space in this manner might be applicable to other MDPs, so for completeness we shortly illustrate how $C$ can be determined for Eq. (2). To this end, we inspect this MDP for the policy that always uses the DB to answer queries. Replacing the minimum in Eq. (2) by this policy yields the equation

$$
\begin{aligned}
g^{D B}+V^{D B}(j, N) & =\lambda_{2} V^{D B}(j+1, N+1)+\mu_{2} V^{D B}(j-1,0) \mathbb{1}_{\{j>0\}} \\
& +\left(1-\lambda_{1}-\lambda_{2}-\mu_{2} \mathbb{1}_{\{j>0\}}\right) V^{D B}(j, N+1) \\
& +\lambda_{1}\left((N-T)^{+}+V^{D B}(j, N+1)\right)
\end{aligned}
$$

where variable $i$ is removed from the notation because it no longer influences the value function. Note that at each increment of $N$ in Eq. (4) costs $\lambda_{1}(N-T)^{+}$are incurred, leading to time-average $\operatorname{costs} g^{D B}$. This suggests that $C:=g^{D B} / \lambda_{1}$ is a suitable constant to replace the $(N-T)^{+}$term in Eq. (2). In [12, Appendix B], we show that $g^{D B}=\lambda_{1} \frac{\left(1-\lambda_{2}\right)^{T+1}}{\lambda_{2}}$ (this can also be obtained via standard queueing theory results), and thus

$$
C=\frac{\left(1-\lambda_{2}\right)^{T+1}}{\lambda_{2}}
$$

\section{$5 \quad$ Near-optimal control policies (Step II)}

We prepare for one-step policy improvement by fixing a policy for the MDP in Eq. (3). For this policy we choose the Bernoulli policy, which randomly assigns incoming queries to either $Q_{1}$ (with probability $\alpha \in[0,1]$ ) or to the DB (with probability $1-\alpha$ ). Replacing the minimum in Eq. (3) by the Bernoulli policy yields the difference equation

$$
\begin{aligned}
\tilde{g}^{\alpha}+\tilde{V}^{\alpha}(i, j) & =\lambda_{2} \tilde{V}^{\alpha}(i, j+1) \\
& +\mu_{1} \tilde{V}^{\alpha}(i-1, j) \mathbb{1}_{\{i>0\}}+\mu_{2} \tilde{V}^{\alpha}(i, j-1) \mathbb{1}_{\{j>0\}} \\
& +\left(1-\lambda_{1}-\lambda_{2}-\mu_{1} \mathbb{1}_{\{i>0\}}-\mu_{2} \mathbb{1}_{\{j>0\}}\right) \tilde{V}^{\alpha}(i, j) \\
& +\lambda_{1} \alpha \cdot\left[\gamma_{1}(i+1)+\gamma_{2} j+\gamma_{3}+\tilde{V}^{\alpha}(i+1, j)\right]+\lambda_{1}(1-\alpha) \cdot\left[C+\tilde{V}^{\alpha}(i, j)\right] .
\end{aligned}
$$

Note how the application of the Bernoulli policy decouples the queueing system from the DB. In the remainder of this section we derive an expression for the relative value function $\tilde{V}^{\alpha}(i, j)$ by solving Eq. (5). This result is summarized in the following theorem:

Theorem 1. The solution to Eq. (5) is given by

$$
\tilde{V}^{\alpha}(i, j)=\frac{\gamma_{1} \lambda_{1} \alpha}{\mu_{1}-\lambda_{1} \alpha} \frac{i(i+1)}{2}+\frac{\gamma_{2} \lambda_{1} \alpha}{\mu_{2}-\lambda_{2}} \frac{j(j+1)}{2},
$$

and

$$
\tilde{g}^{\alpha}=\lambda_{1}(1-\alpha) C+\lambda_{1} \alpha\left(\frac{\gamma_{1} \lambda_{1} \alpha}{\mu_{1}-\lambda_{1} \alpha}+\frac{\gamma_{2} \lambda_{2}}{\mu_{2}-\lambda_{2}}+\gamma_{1}+\gamma_{3}\right) .
$$

Substitution of these expressions for $\tilde{V}^{\alpha}(i, j)$ and $\tilde{g}^{\alpha}$ into Eq. (5) shows that these indeed form a solution. In the following subsections we derive the expressions in Theorem 1 by solving Eq. (5). First, we tackle the inhomogeneous terms $\gamma_{1}(i+1)+\gamma_{2} j+\gamma_{3}$ and $C$ by considering an equation for $\Delta_{1} \tilde{V}^{\alpha}(i, j)=\tilde{V}^{\alpha}(i+1, j)-\tilde{V}^{\alpha}(i, j)$. This removes the inhomogeneous term $C$ and transforms 
the other term to $\gamma_{1}$. Then we look at $\Delta_{1}^{2} \tilde{V}^{\alpha}(i, j)=\Delta_{1} \tilde{V}^{\alpha}(i+1, j)-\Delta_{1} \tilde{V}^{\alpha}(i, j)$, which eliminates the remaining inhomogeneous term $\gamma_{1}$. We solve this equation, and then retrace our steps from $\Delta_{1}^{2} \tilde{V}^{\alpha}(i, j)$ to $\Delta_{1} \tilde{V}^{\alpha}(i, j)$ to $\tilde{V}^{\alpha}(i, j)$.

During the derivation we encounter an issue concerning uniqueness of solutions to the Poisson equation for $\Delta_{1}^{2} \tilde{V}^{\alpha}(i, j)$. There, we postulate a form for a solution and must show that this solution is unique. Showing uniqueness is not trivial and involves several technical arguments that result in additional restrictions on the form of $\Delta_{1}^{2} \tilde{V}^{\alpha}(i, j)$. This important part of the derivation is placed in Appendix A.

\subsection{Solving the difference equation for $\Delta_{1}^{2} \tilde{V}^{\alpha}(i, j)$}

The behavior of the difference equation on the interior of the state space differs from the behavior on the boundaries $\{i=0\}$ and $\{j=0\}$. Therefore, we first study the difference equation for the interior $\{i, j>0\}$. We define $\Delta_{1} \tilde{V}^{\alpha}(i, j)=\tilde{V}^{\alpha}(i+1, j)-\tilde{V}^{\alpha}(i, j)$. For $i>0$ and $j>0$ it holds that

$$
\begin{aligned}
\Delta_{1} \tilde{V}^{\alpha}(i, j) & =\lambda_{1} \alpha\left[\gamma_{1}+\Delta_{1} \tilde{V}^{\alpha}(i+1, j)\right]+\lambda_{1}(1-\alpha) \Delta_{1} \tilde{V}^{\alpha}(i, j)+\lambda_{2} \Delta_{1} \tilde{V}^{\alpha}(i, j+1) \\
& +\mu_{1} \Delta_{1} \tilde{V}^{\alpha}(i-1, j)+\mu_{2} \Delta_{1} \tilde{V}^{\alpha}(i, j-1)+\left(1-\lambda_{1}-\lambda_{2}-\mu_{1}-\mu_{2}\right) \Delta_{1} \tilde{V}^{\alpha}(i, j)
\end{aligned}
$$

Now, define $\Delta_{1}^{2} \tilde{V}^{\alpha}(i, j)=\Delta_{1} \tilde{V}^{\alpha}(i+1, j)-\Delta_{1} \tilde{V}^{\alpha}(i, j)$. With this definition we have that

$$
\begin{aligned}
\Delta_{1}^{2} \tilde{V}^{\alpha}(i, j) & =\lambda_{1} \alpha \Delta_{1}^{2} \tilde{V}^{\alpha}(i+1, j)+\lambda_{1}(1-\alpha) \Delta_{1}^{2} \tilde{V}^{\alpha}(i, j)+\lambda_{2} \Delta_{1}^{2} \tilde{V}^{\alpha}(i, j+1)+\mu_{1} \Delta_{1}^{2} \tilde{V}^{\alpha}(i-1, j) \\
& +\mu_{2} \Delta_{1}^{2} \tilde{V}^{\alpha}(i, j-1)+\left(1-\lambda_{1}-\lambda_{2}-\mu_{1}-\mu_{2}\right) \Delta_{1}^{2} \tilde{V}^{\alpha}(i, j) .
\end{aligned}
$$

We suggestively write this as

$$
\begin{aligned}
\left(\lambda_{1} \alpha+\mu_{1}\right) \Delta_{1}^{2} \tilde{V}^{\alpha}(i, j)+\left(\lambda_{2}+\mu_{2}\right) \Delta_{1}^{2} \tilde{V}^{\alpha}(i, j) & =\lambda_{1} \alpha \Delta_{1}^{2} \tilde{V}^{\alpha}(i+1, j)+\mu_{1} \Delta_{1}^{2} \tilde{V}^{\alpha}(i-1, j) \\
& +\lambda_{2} \Delta_{1}^{2} \tilde{V}^{\alpha}(i, j+1)+\mu_{2} \Delta_{1}^{2} \tilde{V}^{\alpha}(i, j-1) .
\end{aligned}
$$

The notation suggests that the solution to this equation might be split up in a part that only depends on $i$ and a part that only depends on $j$. That is, a solution might be given by $\Delta_{1}^{2} \tilde{V}^{\alpha}(i, j)=$ $\tilde{V}_{1}^{\alpha}(i)+\tilde{V}_{2}^{\alpha}(j)$ with $\tilde{V}_{1}^{\alpha}(i)$ and $\tilde{V}_{2}^{\alpha}(j)$ satisfying

$$
\left\{\begin{array}{c}
\left(\lambda_{1} \alpha+\mu_{1}\right) \tilde{V}_{1}^{\alpha}(i)=\lambda_{1} \alpha \tilde{V}_{1}^{\alpha}(i+1)+\mu_{1} \tilde{V}_{1}^{\alpha}(i-1), \\
\left(\lambda_{2}+\mu_{2}\right) \tilde{V}_{2}^{\alpha}(j)=\lambda_{2} \tilde{V}_{2}^{\alpha}(j+1)+\mu_{2} \tilde{V}_{2}^{\alpha}(j-1) .
\end{array}\right.
$$

These equations are simple homogeneous difference equations of which the solutions are given by

$$
\left\{\begin{array}{l}
\tilde{V}_{1}^{\alpha}(i)=\frac{\mu_{1} \tilde{V}_{1}^{\alpha}(0)-\lambda_{1} \alpha \tilde{V}_{1}^{\alpha}(1)}{\mu_{1}-\lambda_{1} \alpha}+\frac{\lambda_{1} \alpha\left(\tilde{V}_{1}^{\alpha}(1)-\tilde{V}_{1}^{\alpha}(0)\right)\left(\frac{\mu_{1}}{\lambda_{1} \alpha}\right)^{i}}{\mu_{1}-\lambda_{1} \alpha}, \\
\tilde{V}_{2}^{\alpha}(j)=\frac{\mu_{2} \tilde{V}_{2}^{\alpha}(0)-\lambda_{2} \tilde{V}_{2}^{\alpha}(1)}{\mu_{2}-\lambda_{2}}+\frac{\lambda_{2}\left(\tilde{V}_{2}^{\alpha}(1)-\tilde{V}_{2}^{\alpha}(0)\right)\left(\frac{\mu_{2}}{\lambda_{2}}\right)^{j}}{\mu_{2}-\lambda_{2}} .
\end{array}\right.
$$

Note that with these expression for $\tilde{V}_{1} \alpha(i)$ and $\tilde{V}_{2} \alpha(j), \Delta_{1}^{2} \tilde{V}^{\alpha}(i, j)$ is a solution to Eq. (7). It is, however, not immediately obvious that this is also the solution. We return to this issue in Appendix A.

The values for $\tilde{V}_{1}^{\alpha}(0), \tilde{V}_{1}^{\alpha}(1), \tilde{V}_{2}^{\alpha}(0), \tilde{V}_{2}^{\alpha}(1)$ still need to be determined in order to make the solution consistent at the boundaries. For this purpose, consider the boundary $\{j=0\}$. In this case, 
$\Delta_{1} \tilde{V}^{\alpha}(i, 0)$ becomes for $i>0$

$$
\begin{aligned}
\Delta_{1} \tilde{V}^{\alpha}(i, 0) & =\lambda_{1} \alpha\left[\gamma_{1}+\Delta_{1} \tilde{V}^{\alpha}(i+1,0)\right]+\lambda_{1}(1-\alpha) \Delta_{1} \tilde{V}^{\alpha}(i, 0)+\lambda_{2} \Delta_{1} \tilde{V}^{\alpha}(i, 1) \\
& +\mu_{1} \Delta_{1} \tilde{V}^{\alpha}(i-1,0)+\left(1-\lambda_{1}-\lambda_{2}-\mu_{1}\right) \Delta_{1} \tilde{V}^{\alpha}(i, 0) .
\end{aligned}
$$

Similarly, for $\Delta_{1}^{2} \tilde{V}^{\alpha}(i, 0)$ we have that

$$
\begin{aligned}
\Delta_{1}^{2} \tilde{V}^{\alpha}(i, 0) & =\lambda_{1} \alpha \Delta_{1}^{2} \tilde{V}^{\alpha}(i+1,0)+\lambda_{1}(1-\alpha) \Delta_{1}^{2} \tilde{V}^{\alpha}(i, 0)+\lambda_{2} \Delta_{1}^{2} \tilde{V}^{\alpha}(i, 1) \\
& +\mu_{1} \Delta_{1}^{2} \tilde{V}^{\alpha}(i-1,0)+\left(1-\lambda_{1}-\lambda_{2}-\mu_{1}\right) \Delta_{1}^{2} \tilde{V}^{\alpha}(i, 0) .
\end{aligned}
$$

Again, we can suggestively write this as

$$
\begin{aligned}
\left(\lambda_{1} \alpha+\mu_{1}\right) \Delta_{1}^{2} \tilde{V}^{\alpha}(i, 0)+\lambda_{2} \Delta_{1}^{2} \tilde{V}^{\alpha}(i, 0) & =\lambda_{1} \alpha \Delta_{1}^{2} \tilde{V}^{\alpha}(i+1,0)+\mu_{1} \Delta_{1}^{2} \tilde{V}^{\alpha}(i-1,0) \\
& +\lambda_{2} \Delta_{1}^{2} \tilde{V}^{\alpha}(i, 1)
\end{aligned}
$$

leading to the following system of equations

$$
\left\{\begin{aligned}
\left(\lambda_{1} \alpha+\mu_{1}\right) \tilde{V}_{1}^{\alpha}(i) & =\lambda_{1} \alpha \tilde{V}_{1}^{\alpha}(i+1)+\mu_{1} \tilde{V}_{1}^{\alpha}(i-1), \\
\lambda_{2} \tilde{V}_{2}^{\alpha}(0) & =\lambda_{2} \tilde{V}_{2}^{\alpha}(1) .
\end{aligned}\right.
$$

From these expressions, we obtain that on the boundary $\{j=0\}$, the MDP behaves exactly the same as the MDP on the interior. Furthermore, it shows that $\tilde{V}_{2}^{\alpha}(0)=\tilde{V}_{2}^{\alpha}(1)$ and thus that $\tilde{V}_{2}^{\alpha}(j)$ in Eq. (9) is a constant: $\tilde{V}_{2}^{\alpha}(j)=c_{2}$. Without loss of generality, we can set $c_{2}=0$ and determine $\Delta_{1}^{2} \tilde{V}^{\alpha}(i, j)$ completely from $\tilde{V}_{1}^{\alpha}(i)$. Summarizing, this gives us the result that $\Delta_{1}^{2} \tilde{V}^{\alpha}(i, j)=\tilde{V}_{1}^{\alpha}(i)+\tilde{V}_{2}^{\alpha}(j)$, where $\tilde{V}_{2}^{\alpha}(j) \equiv 0$ and

$$
\tilde{V}_{1}^{\alpha}(i)=\frac{\mu_{1} \tilde{V}_{1}^{\alpha}(0)-\lambda_{1} \alpha \tilde{V}_{1}^{\alpha}(1)}{\mu_{1}-\lambda_{1} \alpha}+\frac{\lambda_{1} \alpha\left(\tilde{V}_{1}^{\alpha}(1)-\tilde{V}_{1}^{\alpha}(0)\right)\left(\frac{\mu_{1}}{\lambda_{1} \alpha}\right)^{i}}{\mu_{1}-\lambda_{1} \alpha} .
$$

\subsection{Analyzing $\Delta_{1} \tilde{V}^{\alpha}(i, j+1)-\Delta_{1} \tilde{V}^{\alpha}(i, j)$}

For the derivation of an expression for $\tilde{V}^{\alpha}(i, j)$ (which we do in the next sections), we require the following intermediate result:

Lemma 1. The relative value function $\tilde{V}^{\alpha}(i, j)$ satisfies

$$
\Delta_{2} \Delta_{1} \tilde{V}^{\alpha}(i, j)=0
$$

where $\Delta_{2} \Delta_{1} \tilde{V}^{\alpha}(i, j):=\Delta_{1} \tilde{V}^{\alpha}(i, j+1)-\Delta_{1} \tilde{V}^{\alpha}(i, j)$

In words, Lemma 1 states that first differencing $\tilde{V}^{\alpha}(i, j)$ in $i$, followed by differencing the result in $j$, equals 0 .

Proof. We start again for the interior $\{i, j>0\}$, where we have the following relation for $i>0$ and 
$j>0$ :

$$
\begin{aligned}
\Delta_{1} \tilde{V}^{\alpha}(i, j) & =\lambda_{1} \alpha\left[\gamma_{1}+\Delta_{1} \tilde{V}^{\alpha}(i+1, j)\right]+\lambda_{1}(1-\alpha) \Delta_{1} \tilde{V}^{\alpha}(i, j) \\
& +\lambda_{2} \Delta_{1} \tilde{V}^{\alpha}(i, j+1)+\mu_{1} \Delta_{1} \tilde{V}^{\alpha}(i-1, j)+\mu_{2} \Delta_{1} \tilde{V}^{\alpha}(i, j-1) \\
& +\left(1-\lambda_{1}-\lambda_{2}-\mu_{1}-\mu_{2}\right) \Delta_{1} \tilde{V}^{\alpha}(i, j) .
\end{aligned}
$$

We find for $\Delta_{2} \Delta_{1} \tilde{V}^{\alpha}(i, j)$

$$
\begin{aligned}
\Delta_{2} \Delta_{1} \tilde{V}^{\alpha}(i, j) & =\lambda_{1} \alpha \Delta_{2} \Delta_{1} \tilde{V}^{\alpha}(i+1, j)+\lambda_{1}(1-\alpha) \Delta_{2} \Delta_{1} \tilde{V}^{\alpha}(i, j) \\
& +\lambda_{2} \Delta_{2} \Delta_{1} \tilde{V}^{\alpha}(i, j+1)+\mu_{1} \Delta_{2} \Delta_{1} \tilde{V}^{\alpha}(i-1, j)+\mu_{2} \Delta_{2} \Delta_{1} \tilde{V}^{\alpha}(i, j-1) \\
& +\left(1-\lambda_{1}-\lambda_{2}-\mu_{1}-\mu_{2}\right) \Delta_{2} \Delta_{1} \tilde{V}^{\alpha}(i, j) .
\end{aligned}
$$

By similar line of reasoning as before, we derive that $\Delta_{2} \Delta_{1} \tilde{V}^{\alpha}(i, j)=\bar{V}_{1}(i)+\bar{V}_{2}(j)$, with

$$
\begin{aligned}
& \bar{V}_{1}(i)=\frac{\mu_{1} \bar{V}_{1}(0)-\lambda_{1} \alpha \bar{V}_{1}(1)}{\mu_{1}-\lambda_{1} \alpha}+\frac{\lambda_{1} \alpha\left(\bar{V}_{1}(1)-\bar{V}_{1}(0)\right)\left(\frac{\mu_{1}}{\lambda_{1} \alpha}\right)^{i}}{\mu_{1}-\lambda_{1} \alpha}, \\
& \bar{V}_{2}(j)=\frac{\mu_{2} \bar{V}_{2}(0)-\lambda_{2} \bar{V}_{2}(1)}{\mu_{2}-\lambda_{2}}+\frac{\lambda_{2}\left(\bar{V}_{2}(1)-\bar{V}_{2}(0)\right)\left(\frac{\mu_{2}}{\lambda_{2}}\right)^{j}}{\mu_{2}-\lambda_{2}},
\end{aligned}
$$

where $\bar{V}_{1}(0), \bar{V}_{1}(1), \bar{V}_{2}(0), \bar{V}_{2}(1)$ are determined from $\Delta_{2} \Delta_{1} \tilde{V}^{\alpha}(i, 0)$ and $\Delta_{2} \Delta_{1} \tilde{V}^{\alpha}(0, j)$. We start with the former by inspecting the term $\Delta_{1} \tilde{V}^{\alpha}(i, 1)$. From Expression (6) we have that

$$
\begin{aligned}
\Delta_{1} \tilde{V}^{\alpha}(i, 1) & =\lambda_{1} \alpha\left[\gamma_{1}+\Delta_{1} \tilde{V}^{\alpha}(i+1,1)\right]+\lambda_{1}(1-\alpha) \Delta_{1} \tilde{V}^{\alpha}(i, 1) \\
& +\lambda_{2} \Delta_{1} \tilde{V}^{\alpha}(i, 2)+\mu_{1} \Delta_{1} \tilde{V}^{\alpha}(i-1,1)+\mu_{2} \Delta_{1} \tilde{V}^{\alpha}(i, 0) \\
& +\left(1-\lambda_{1}-\lambda_{2}-\mu_{1}-\mu_{2}\right) \Delta_{1} \tilde{V}^{\alpha}(i, 1) .
\end{aligned}
$$

For the term $\Delta_{1} \tilde{V}^{\alpha}(i, 0)$ we derive from Expression (10) that

$$
\begin{aligned}
\Delta_{1} \tilde{V}^{\alpha}(i, 0) & =\lambda_{1} \alpha\left[\gamma_{1}+\Delta_{1} \tilde{V}^{\alpha}(i+1,0)\right]+\lambda_{1}(1-\alpha) \Delta_{1} \tilde{V}^{\alpha}(i, 0)+\lambda_{2} \Delta_{1} \tilde{V}^{\alpha}(i, 1) \\
& +\mu_{1} \Delta_{1} \tilde{V}^{\alpha}(i-1,0)+\left(1-\lambda_{1}-\lambda_{2}-\mu_{1}\right) \Delta_{1} \tilde{V}^{\alpha}(i, 0) .
\end{aligned}
$$

Consequently

$$
\begin{aligned}
\Delta_{2} \Delta_{1} \tilde{V}^{\alpha}(i, 0) & =\lambda_{1} \alpha \Delta_{2} \Delta_{1} \tilde{V}^{\alpha}(i+1,0)+\lambda_{1}(1-\alpha) \Delta_{2} \Delta_{1} \tilde{V}^{\alpha}(i, 0)+\lambda_{2} \Delta_{2} \Delta_{1} \tilde{V}^{\alpha}(i, 1) \\
& +\mu_{1} \Delta_{2} \Delta_{1} \tilde{V}^{\alpha}(i-1,0)-\mu_{2} \Delta_{2} \Delta_{1} \tilde{V}^{\alpha}(i, 0)+\left(1-\lambda_{1}-\lambda_{2}-\mu_{1}\right) \Delta_{2} \Delta_{1} \tilde{V}^{\alpha}(i, 0),
\end{aligned}
$$

which reduces to

$$
\begin{aligned}
& \left(\lambda_{2}+\mu_{2}\right) \Delta_{2} \Delta_{1} \tilde{V}^{\alpha}(i, 0)+\left(\lambda_{1} \alpha+\mu_{1}\right) \Delta_{2} \Delta_{1} \tilde{V}^{\alpha}(i, 0)= \\
& \lambda_{2} \Delta_{2} \Delta_{1} \tilde{V}^{\alpha}(i, 1)+\lambda_{1} \alpha \Delta_{2} \Delta_{1} \tilde{V}^{\alpha}(i+1,0)+\mu_{1} \Delta_{2} \Delta_{1} \tilde{V}^{\alpha}(i-1,0) .
\end{aligned}
$$

A solution can be obtained by splitting the equation into a solution of the type $\Delta_{2} \Delta_{1} \tilde{V}^{\alpha}(i, j)=$ $\bar{V}_{1}(i)+\bar{V}_{2}(j)$, resulting in

$$
\left\{\begin{aligned}
\left(\lambda_{2}+\mu_{2}\right) \Delta_{2} \Delta_{1} \tilde{V}^{\alpha}(i, 0) & =\lambda_{2} \Delta_{2} \Delta_{1} \tilde{V}^{\alpha}(i, 1), \\
\left(\lambda_{1} \alpha+\mu_{1}\right) \Delta_{2} \Delta_{1} \tilde{V}^{\alpha}(i, 0) & =\lambda_{1} \alpha \Delta_{2} \Delta_{1} \tilde{V}^{\alpha}(i+1,0)+\mu_{1} \Delta_{2} \Delta_{1} \tilde{V}^{\alpha}(i-1,0) .
\end{aligned}\right.
$$


The upper equation translates to

$$
\left(\lambda_{2}+\mu_{2}\right)\left(\bar{V}_{1}(i)+\bar{V}_{2}(0)\right)=\lambda_{2}\left(\bar{V}_{1}(i)+\bar{V}_{2}(1)\right)
$$

or

$$
\mu_{2} \bar{V}_{1}(i)=\lambda_{2} \bar{V}_{2}(1)-\left(\lambda_{2}+\mu_{2}\right) \bar{V}_{2}(0) .
$$

So $\bar{V}_{1}(i)$ is constant for $i>0$, which we denote by $\bar{V}_{1}(i)=\bar{c}_{1}$. By repeating the arguments above for the boundary $\{i=0\}$, we find that $\bar{V}_{2}(j):=\bar{c}_{2}$ is constant. As a consequence, Eq. (15) reduces to

$$
\mu_{2} \bar{c}_{1}=\lambda_{2} \bar{c}_{2}-\left(\lambda_{2}+\mu_{2}\right) \bar{c}_{2}
$$

or

$$
\mu_{2} \bar{c}_{1}=-\mu_{2} \bar{c}_{2}
$$

i.e., $\bar{c}_{1}=-\bar{c}_{2}$ and thus $\Delta_{2} \Delta_{1} \tilde{V}^{\alpha}(i, j)=0$, which concludes the proof.

\subsection{Solving the difference equation for $\Delta_{1} \tilde{V}^{\alpha}(i, j)$}

So far, we have found that $\Delta_{1}^{2} \tilde{V}^{\alpha}(i, j)$ satisfies

$$
\Delta_{1}^{2} \tilde{V}^{\alpha}(i, j)=\frac{\mu_{1} \tilde{V}_{1}^{\alpha}(0)-\lambda_{1} \alpha \tilde{V}_{1}^{\alpha}(1)}{\mu_{1}-\lambda_{1} \alpha}+\frac{\lambda_{1} \alpha\left(\tilde{V}_{1}^{\alpha}(1)-\tilde{V}_{1}^{\alpha}(0)\right)\left(\frac{\mu_{1}}{\lambda_{1} \alpha}\right)^{i}}{\mu_{1}-\lambda_{1} \alpha},
$$

and we have proved that $\Delta_{2} \Delta_{1} \tilde{V}^{\alpha}(i, j)=0$ in Lemma 1 . Note that this implies that $\Delta_{1} \tilde{V}^{\alpha}(i, j)$ is independent of $j$ for all $i$. We continue the proof of Thm. 1 by reverting the differencing in $i$ used to obtain Eq. (16).

Recall that $\Delta_{1}^{2} \tilde{V}^{\alpha}(i, j)=\Delta_{1} \tilde{V}^{\alpha}(i+1, j)-\Delta_{1} \tilde{V}^{\alpha}(i, j)$. By summing over $i$, and then using the right-hand side of Eq. (16), we can get an expression for $\Delta_{1} \tilde{V}^{\alpha}(i, j)$ :

$$
\begin{aligned}
\Delta_{1} \tilde{V}^{\alpha}(i, j) & =\Delta_{1} \tilde{V}^{\alpha}(0, j)+\sum_{k=0}^{i-1} \Delta_{1}^{2} \tilde{V}^{\alpha}(k, j) \\
& =\Delta_{1} \tilde{V}^{\alpha}(0, j)+\frac{\mu_{1} \tilde{V}_{1}^{\alpha}(0)-\lambda_{1} \alpha \tilde{V}_{1}^{\alpha}(1)}{\mu_{1}-\lambda_{1} \alpha} i+\frac{\lambda_{1} \alpha\left(\tilde{V}_{1}^{\alpha}(1)-\tilde{V}_{1}^{\alpha}(0)\right)}{\mu_{1}-\lambda_{1} \alpha} \frac{1-\left(\frac{\mu_{1}}{\lambda_{1} \alpha}\right)^{i}}{1-\frac{\mu_{1}}{\lambda_{1} \alpha}} .
\end{aligned}
$$

Here, $\Delta_{1} \tilde{V}^{\alpha}(0, j)$ is a constant (by Lemma 1) which we determine below. Substituting the expression for $\Delta_{1} \tilde{V}^{\alpha}(i, j)$ from Eq. (17) into Eq. (6), we find that necessarily

$$
\mu_{1} \tilde{V}_{1}^{\alpha}(0)-\lambda_{1} \alpha \tilde{V}_{1}^{\alpha}(1)=\gamma_{1} \lambda_{1} \alpha .
$$

Solving this for $\tilde{V}_{1}^{\alpha}(1)$ and substituting the result into Eq. (16) yields

$$
\Delta_{1}^{2} \tilde{V}^{\alpha}(i, j)=\frac{\gamma_{1} \lambda_{1} \alpha}{\mu_{1}-\lambda_{1} \alpha}+\left[\tilde{V}_{1}^{\alpha}(0)-\frac{\gamma_{1} \lambda_{1} \alpha}{\mu_{1}-\lambda_{1} \alpha}\right]\left(\frac{\mu_{1}}{\lambda_{1} \alpha}\right)^{i} .
$$


Hence, $\Delta_{1} \tilde{V}^{\alpha}(i, j)$ becomes

$$
\Delta_{1} \tilde{V}^{\alpha}(i, j)=\Delta_{1} \tilde{V}^{\alpha}(0, j)+\frac{\gamma_{1} \lambda_{1} \alpha}{\mu_{1}-\lambda_{1} \alpha} i+\left[\tilde{V}_{1}^{\alpha}(0)-\frac{\gamma_{1} \lambda_{1} \alpha}{\mu_{1}-\lambda_{1} \alpha}\right] \frac{1-\left(\frac{\mu_{1}}{\lambda_{1} \alpha}\right)^{i}}{1-\frac{\mu_{1}}{\lambda_{1} \alpha}} .
$$

Now we turn our attention to determining the (constant) $\Delta_{1} \tilde{V}^{\alpha}(0, j)$ by inspecting the corresponding difference equation:

$$
\begin{aligned}
\Delta_{1} \tilde{V}^{\alpha}(0, j) & =\lambda_{1} \alpha\left[\gamma_{1}+\Delta_{1} \tilde{V}^{\alpha}(1, j)\right]+\lambda_{1}(1-\alpha) \Delta_{1} \tilde{V}^{\alpha}(0, j)+\lambda_{2} \Delta_{1} \tilde{V}^{\alpha}(0, j+1) \\
& +\mu_{2} \Delta_{1} \tilde{V}^{\alpha}(0, j-1)+\left(1-\lambda_{1}-\lambda_{2}-\mu_{1}-\mu_{2}\right) \Delta_{1} \tilde{V}^{\alpha}(0, j) .
\end{aligned}
$$

We can rewrite this equation as follows:

$$
\begin{aligned}
0 & =\lambda_{2}\left[\Delta_{1} \tilde{V}^{\alpha}(0, j+1)-\Delta_{1} \tilde{V}^{\alpha}(0, j)\right]+\lambda_{1} \alpha\left[\Delta_{1} \tilde{V}^{\alpha}(1, j)-\Delta_{1} \tilde{V}^{\alpha}(0, j)\right] \\
& +\gamma_{1} \lambda_{1} \alpha+\mu_{2}\left[\Delta_{1} \tilde{V}^{\alpha}(0, j-1)-\Delta_{1} \tilde{V}^{\alpha}(0, j)\right]-\mu_{1} \Delta_{1} \tilde{V}^{\alpha}(0, j) .
\end{aligned}
$$

Using Lemma 1 we find

$$
0=\lambda_{1} \alpha\left[\Delta_{1} \tilde{V}^{\alpha}(1, j)-\Delta_{1} \tilde{V}^{\alpha}(0, j)\right]+\gamma_{1} \lambda_{1} \alpha-\mu_{1} \Delta_{1} \tilde{V}^{\alpha}(0, j) .
$$

Eq. (18) tells us that $\Delta_{1} \tilde{V}^{\alpha}(1, j)=\Delta_{1} \tilde{V}^{\alpha}(0, j)+\tilde{V}_{1}^{\alpha}(0)$, so

$$
\Delta_{1} \tilde{V}^{\alpha}(0, j)=\frac{\lambda_{1} \alpha}{\mu_{1}} \tilde{V}_{1}^{\alpha}(0)+\frac{\gamma_{1} \lambda_{1} \alpha}{\mu_{1}} .
$$

Substitution into Eq. (18) yields

$$
\Delta_{1} \tilde{V}^{\alpha}(i, j)=\frac{\lambda_{1} \alpha}{\mu_{1}} \tilde{V}_{1}^{\alpha}(0)+\frac{\gamma_{1} \lambda_{1} \alpha}{\mu_{1}}+\frac{\gamma_{1} \lambda_{1} \alpha}{\mu_{1}-\lambda_{1} \alpha} i+\left[\tilde{V}_{1}^{\alpha}(0)-\frac{\gamma_{1} \lambda_{1} \alpha}{\mu_{1}-\lambda_{1} \alpha}\right] \frac{1-\left(\frac{\mu_{1}}{\lambda_{1} \alpha}\right)^{i}}{1-\frac{\mu_{1}}{\lambda_{1} \alpha}}
$$

\subsection{Deriving $\tilde{V}^{\alpha}(i, j)$}

We derive an expression for $\tilde{V}^{\alpha}(i, j)$ by using $\Delta_{1} \tilde{V}^{\alpha}(i, j)=\tilde{V}^{\alpha}(i+1, j)-\tilde{V}^{\alpha}(i, j)$, summing over $i$, and then using Eq. (19):

$$
\begin{aligned}
\tilde{V}^{\alpha}(i, j) & =\tilde{V}^{\alpha}(0, j)+\sum_{k=0}^{i-1} \Delta_{1} \tilde{V}^{\alpha}(k, j) \\
& =\tilde{V}^{\alpha}(0, j)+i \cdot\left(\frac{\lambda_{1} \alpha}{\mu_{1}} \tilde{V}_{1}^{\alpha}(0)+\frac{\gamma_{1} \lambda_{1} \alpha}{\mu_{1}}\right)+\frac{\gamma_{1} \lambda_{1} \alpha}{\mu_{1}-\lambda_{1} \alpha} \frac{i(i-1)}{2} \\
& +\frac{1}{1-\frac{\mu_{1}}{\lambda_{1} \alpha}}\left[\tilde{V}_{1}^{\alpha}(0)-\frac{\gamma_{1} \lambda_{1} \alpha}{\mu_{1}-\lambda_{1} \alpha}\right]\left[i-\frac{1-\left(\frac{\mu_{1}}{\lambda_{1} \alpha}\right)^{i}}{1-\frac{\mu_{1}}{\lambda_{1} \alpha}}\right] .
\end{aligned}
$$

In the derivation so far we have postulated a form of a solution several times (Eqs. (8) and (11$14)$ ), resulting in the expression for $\tilde{V}^{\alpha}(i, j)$ in Eq. (20). Here, we finally deal with the uniqueness issue. As mentioned earlier, ensuring uniqueness of a solution $\tilde{V}^{\alpha}(i, j)$ to Eq. (5) is not trivial. Conventional uniqueness proofs rely on bounded cost functions, and the cost function in Eq. (1) is unbounded. Addressing this point requires several technical arguments which we, for readability, place in Appendix A. In short, uniqueness is ensured if $\tilde{V}^{\alpha}(i, j)$ does not grow exponentially fast. Therefore, we choose the remaining constant $\tilde{V}_{1}^{\alpha}(0)$ in Eq. (20) such that the exponential term $\left(\frac{\mu_{1}}{\lambda_{1} \alpha}\right)^{i}$ 
disappears:

$$
\tilde{V}_{1}^{\alpha}(0)=\frac{\gamma_{1} \lambda_{1} \alpha}{\mu_{1}-\lambda_{1} \alpha}
$$

Substitution into Eq. (20) yields

$$
\tilde{V}^{\alpha}(i, j)=\tilde{V}^{\alpha}(0, j)+\frac{\gamma_{1} \lambda_{1} \alpha}{\mu_{1}-\lambda_{1} \alpha} i+\frac{\gamma_{1} \lambda_{1} \alpha}{\mu_{1}-\lambda_{1} \alpha} \frac{i(i-1)}{2},
$$

or

$$
\tilde{V}^{\alpha}(i, j)=\tilde{V}^{\alpha}(0, j)+\frac{\gamma_{1} \lambda_{1} \alpha}{\mu_{1}-\lambda_{1} \alpha} \frac{i(i+1)}{2} .
$$

Repeating the steps in Sections 5.1-5.4 for differencing in $j$ instead of $i$ gives

$$
\tilde{V}^{\alpha}(i, j)=\tilde{V}^{\alpha}(i, 0)+\frac{\gamma_{2} \lambda_{1} \alpha}{\mu_{2}-\lambda_{2}} \frac{j(j+1)}{2},
$$

so that necessarily

$$
\tilde{V}^{\alpha}(i, j)=\frac{\gamma_{1} \lambda_{1} \alpha}{\mu_{1}-\lambda_{1} \alpha} \frac{i(i+1)}{2}+\frac{\gamma_{2} \lambda_{1} \alpha}{\mu_{2}-\lambda_{2}} \frac{j(j+1)}{2} .
$$

Finally, substituting this expression for $\tilde{V}^{\alpha}(i, j)$ into Eq. (5) and solving for $\tilde{g}^{\alpha}$ yields

$$
\tilde{g}^{\alpha}=\lambda_{1}(1-\alpha) C+\lambda_{1} \alpha\left(\frac{\gamma_{1} \lambda_{1} \alpha}{\mu_{1}-\lambda_{1} \alpha}+\frac{\gamma_{2} \lambda_{2}}{\mu_{2}-\lambda_{2}}+\gamma_{1}+\gamma_{3}\right) .
$$

This concludes the derivation of the expressions in Theorem 1.

Remark: The structure of $\tilde{V}^{\alpha}(i, j)$ in Eq. (21) and $\tilde{g}^{\alpha}$ in Eq. (22) can be explained intuitively using known results about the $M / M / 1$ queue. The Bernoulli policy chooses $Q_{1}$ with probability $\alpha$ and the DB with probability $1-\alpha$, thereby decoupling the system in three separate elements: the $\mathrm{DB}, Q_{1}$, and $Q_{2}$. Choosing the DB incurs a penalty $C$, which results in time-average costs $\lambda_{1}(1-\alpha) C$. This corresponds to the first term in Eq. (22). The alternative choice (assignment to the queueing system) incurs costs $\gamma_{1}(i+1)+\gamma_{2} j+\gamma_{3}$. Note that the two queues (the first with arrival rate $\lambda_{1} \alpha$, the second with arrival rate $\lambda_{2}$ ) are independent and that the $i$ and $j$ terms are summed in the cost function. Consequently, the time-average costs of assignment to the queueing system are just the summed time-average costs of the two $M / M / 1$ queues with holding costs $\gamma_{1}$ and $\gamma_{2}$ respectively (and of fixed costs $\gamma_{1}+\gamma_{3}$ ). For a $M / M / 1$ queue we know (see [6]) that $g=\frac{\rho}{1-\rho} h$, with $\rho=\lambda / \mu$ the system load, $\lambda$ the arrival rate, $\mu$ the service rate, and holding costs $h$. This explains the $\frac{\gamma_{1} \lambda_{1} \alpha}{\mu_{1}-\lambda_{1} \alpha}+\frac{\gamma_{2} \lambda_{2}}{\mu_{2}-\lambda_{2}}+\gamma_{1}+\gamma_{3}$ term (multiplied by $\lambda_{1} \alpha$ ) in Eq. (22). Also, the value function $\tilde{V}^{\alpha}(i, j)$ in Eq. (21) is just the sum of the value functions of the two $M / M / 1$ queues (multiplied by $\lambda_{1} \alpha$ ). 


\section{One-step policy improvement (Step III)}

\subsection{Obtaining the improved policy}

In the previous section we approximated $V(i, j, N)$ by $\tilde{V}^{\alpha}(i, j)$. Now we apply one-step policy improvement by inspecting the minimization term in Eq. (2), with $V(i, j, N)$ replaced by $\tilde{V}^{\alpha}(i, j)$ :

$$
\min \left\{\gamma_{1}(i+1)+\gamma_{2} j+\gamma_{3}+\tilde{V}^{\alpha}(i+1, j) ;(N-T)^{+}+\tilde{V}^{\alpha}(i, j)\right\} .
$$

Hence, the improved policy assigns a query to the DB if

$$
\begin{array}{rlrl}
\gamma_{1}(i+1)+\gamma_{2} j+\gamma_{3}+\tilde{V}^{\alpha}(i+1, j) & \geq(N-T)^{+}+\tilde{V}^{\alpha}(i, j) & & \Leftrightarrow \\
\gamma_{1}(i+1)+\gamma_{2} j+\gamma_{3}+\frac{\gamma_{1} \lambda_{1} \alpha}{\mu_{1}-\lambda_{1} \alpha} \frac{(i+1)(i+2)}{2} & \geq(N-T)^{+}+\frac{\gamma_{1} \lambda_{1} \alpha}{\mu_{1}-\lambda_{1} \alpha} \frac{i(i+1)}{2} & \Leftrightarrow \\
\gamma_{1}(i+1)+\gamma_{2} j+\gamma_{3}+\frac{\gamma_{1} \lambda_{1} \alpha}{\mu_{1}-\lambda_{1} \alpha}(i+1) & \geq(N-T)^{+} & \Leftrightarrow \\
\frac{\gamma_{1} \mu_{1}}{\mu_{1}-\lambda_{1} \alpha}(i+1)+\gamma_{2} j+\gamma_{3} & \geq(N-T)^{+} & \Leftrightarrow \\
\frac{\gamma_{1}}{1-\rho_{1} \alpha}(i+1)+\gamma_{2} j+\gamma_{3} & \geq(N-T)^{+} . & \Leftrightarrow
\end{array}
$$

Note that this improved policy is independent of the constant $C$, as mentioned at the beginning of Section 5. Also, in the derivation of Eq. (24) we see that by choosing $\gamma_{1}(i+1)$ rather than $\gamma_{1} i$ in the cost function, we obtain an expression where the $\alpha$ only occurs in front of the $(i+1)$ term. This allows us to intuitively explain the role of $\alpha$ : it acts as a tuning parameter of the improved policy, determining the influence of the number of queries $i$ in the system on the decisions. For $\alpha=0$ the improved policy is independent of $\lambda_{1}$, but as $\alpha$ gets closer to 1 the number of queries in the system is weighed more heavily in the decision, and the policy becomes more biased towards the DB.

\subsection{Determining $\alpha$}

The improved policy in Eq. (24) specifies a class of policies - only after choosing $\alpha$ (originally the parameter of the Bernoulli policy) do we have a concrete policy for which we can, e.g., determine average costs. However, we have no analytical relationship between $V(i, j, N)$ and $\tilde{V}^{\alpha}(i, j)$, and thus determining $\alpha$ analytically is not possible. The best analytical option we have is to minimize $\tilde{g}^{\alpha}$ (of the Bernoulli policy applied to the simplified MDP) w.r.t. $\alpha$, and use the resulting minimum for the improved policy. Unfortunately, subsequent experiments with value iteration show unsatisfactory performance of the resulting improved policy. We observed this behavior for various values for $\lambda_{1}, \lambda_{2}, \mu_{1}, \mu_{2}$, and $T$, so the unsatisfactory performance was general. The $\tilde{V}^{\alpha}(i, j)$ and $\tilde{g}^{\alpha}$ do not approximate $V(i, j, N)$ and $g$ from Eq. (2) sufficiently well.

Fortunately, a simple numerical approach allows us to compute an $\alpha$ that yields an improved policy with the desired near-optimal performance. To illustrate this procedure, we start by looking at Fig. 4, which shows approximations of the average costs $g^{\prime}$ of the improved policy (obtained with value iteration) as a function of $\alpha$. The shape resembles a second-degree polynomial, and by carefully fitting such a polynomial to the approximate values, we can approximate $g^{\prime}(\alpha)$. Then, we use the minimum $\hat{\alpha}$ of the fitted polynomial as input for the improved policy. Note that, due to this procedure, the improved policy is not an analytical policy: every time an improved policy is required, $\hat{\alpha}$ must be computed using the fitting procedure. 
This approach for determining $\hat{\alpha}$ requires several approximate values $\alpha_{i}$ that together capture the shape of $g^{\prime}(\alpha)$. They should be positioned such that the minimum of the polynomial and that of $g^{\prime}(\alpha)$ are at approximately the same $\alpha$-value. Strictly speaking we need only three $\alpha$-values to fit a second-degree polynomial. However, $g^{\prime}(\alpha)$ is not truly a second-degree polynomial, and using four values results in a more appropriate fit in cases where $g^{\prime}(\alpha)$ resembles the polynomial shape less. So how should we position these four points? In the next section we argue that the most interesting scenario is one where $\rho_{1}$ is large. In this scenario, the number of queries $i$ in the system is typically large. Recall that $\hat{\alpha}$ influences the improved policy in Eq. (24) via $i$ : as $\hat{\alpha}$ gets closer to 1 the number of queries in the system is weighed more heavily in the decision, and the policy becomes more biased towards the DB. Hence, we should concentrate the fit of the polynomial on the right side of the interval, near $\alpha=1$. Following this reasoning, we take $\alpha_{1}=0.25, \alpha_{2}=0.6, \alpha_{3}=0.85$, and $\alpha_{4}=0.95$.

The value of each $g^{\prime}\left(\alpha_{i}\right)$ is obtained by running value iteration. The time needed to execute these four runs of value iteration should be shorter than the time needed to compute the optimal policy, otherwise there is no reason to use the improved policy. To this end, we do value iteration for the $g^{\prime}\left(\alpha_{i}\right)$ on a much smaller state space than the one used for finding the optimal policy. Suppose that we run value iteration for the optimal policy on the truncated state space $\overline{\mathcal{X}}=\left[0, K_{1}\right] \times\left[0, K_{2}\right] \times\left[0, K_{3}\right]$ (in Section 7 we determine $K_{1}, K_{2}$, and $K_{3}$ experimentally in such a way that we avoid boundary effects). For the $g^{\prime}\left(\alpha_{i}\right)$, we use the further truncated state space $\hat{\mathcal{X}}:=\left[0,\left\lfloor\frac{K_{1}}{4}\right\rfloor\right] \times\left[0,\left\lfloor\frac{K_{2}}{4}\right\rfloor\right] \times$ $\left[0,\left\lfloor\frac{K_{3}}{4}\right\rfloor\right]$. This effectively reduces the time needed to calculate $\hat{\alpha}$ (and thus also the improved policy) to a mere fraction of the time needed to obtain an optimal policy. The number by which the $K_{i}$ are divided (4) is determined experimentally to yield both low time-average costs and a short run time for the improved policy. Note that the further reduction of the state space is appropriate, because we do not require numerically accurate approximations of $g^{\prime}\left(\alpha_{1}\right), \ldots, g^{\prime}\left(\alpha_{4}\right)$. We only need to capture the general shape illustrated in Fig. 4.

The complete procedure is as follows:

1. Calculate the bounds of the further truncated state space $\hat{\mathcal{X}}$.

2. For each of the values $\alpha_{i}$, evaluate the improved policy using $\hat{\mathcal{X}}$ as state space, and record $g^{\prime}\left(\alpha_{i}\right)$.

3. Fit a second degree polynomial through $g^{\prime}\left(\alpha_{1}\right), \ldots, g^{\prime}\left(\alpha_{4}\right)$ using least squares.

4. Calculate the minimum of this polynomial, and use the $\alpha$-value for which this minimum is attained as $\hat{\alpha}$.

In the example in Fig. 4 this procedure yields $\hat{\alpha}=0.48$, which agrees well with what the figure suggests. Fig. 4 is generated with parameters corresponding to a high load on $Q_{1}$ and low load on $Q_{2}\left(\mu_{1}=\mu_{2}=0.3, T=2, \gamma_{1}=\gamma_{2}=\gamma_{3}=3, \rho_{1}=0.8, \rho_{2}=0.1\right)$. We expect a significant fraction of the queries to be assigned to $Q_{1}$, since a low load on $Q_{2}$ results in large $N$ and thus using the DB is expensive. This observation is supported by the value $\hat{\alpha}=0.48$ that our procedure yields for the improved policy. Also, the figure indicates that the sensitivity of the average $\operatorname{costs} g^{\prime}(\alpha)$ to $\alpha$ is minor around the minimum $\hat{\alpha}$. 


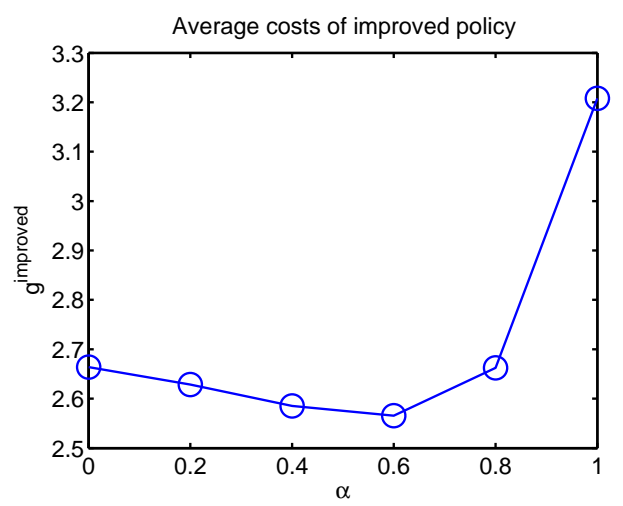

Figure 4. Average costs $g^{\prime}$ of the improved policy, for various values of $\alpha$. The points in the graph are obtained with value iteration, using parameters $\mu_{1}=\mu_{2}=0.3, T=2, \gamma_{1}=\gamma_{2}=\gamma_{3}=3, \rho_{1}=0.8, \rho_{2}=0.1$. Our fitting approach for determining the minimum $\hat{\alpha}$ yields $\hat{\alpha}=0.48$

\section{$7 \quad$ Numerical results}

In this section we experimentally inspect the performance of the improved policy by numerically comparing it to the optimal policy. Additionally, we compare a traditional age-threshold policy and a myopic policy to the optimal policy, allowing us to assess how the improved policy performs in relation to these other two policies. The three policies that we compare to the optimal policy are given by

$$
\begin{aligned}
& \pi^{\text {threshold }}(i, j, N)= \begin{cases}\mathrm{DB}, & \text { if } N \leq T, \\
Q_{1}, & \text { otherwise }\end{cases} \\
& \pi^{\text {myopic }}(i, j, N)= \begin{cases}\mathrm{DB}, & \text { if } \gamma_{1}(i+1)+\gamma_{2} j+\gamma_{3} \geq(N-T)^{+}, \\
Q_{1}, & \text { otherwise. }\end{cases} \\
& \pi^{\prime}(i, j, N)= \begin{cases}\mathrm{DB}, & \text { if } \frac{\gamma_{1}}{1-\rho_{1} \hat{\alpha}}(i+1)+\gamma_{2} j+\gamma_{3} \geq(N-T)^{+}, \\
Q_{1}, & \text { otherwise. }\end{cases}
\end{aligned}
$$

Looking at the three policies, we see that the age-threshold policy ignores the load on the queueing system, and bases its actions solely on the age $N$. The myopic policy takes the load of the system into account, by assigning queries to the DB or $Q_{1}$ based on the cost function in Eq. (1) only, ignoring the value function $V(i, j, N)$. In contrast, the improved policy is based on an approximation of the value function, and thus does include expectations about future query arrivals and report requests in its decisions. These expectations are captured by the parameter $\hat{\alpha}$, which determines how much emphasis the improved policy puts on the number of queries $i$ in the system. Note that for $\hat{\alpha}=0$ the improved and myopic policy are identical.

Looking at our scenario, we expect that as $\rho_{2} \rightarrow 1$, performance should be quite good, since the DB is refreshed often and thus most queries can be answered from the DB. Additionally, in situations with small $\rho_{1}$ the controller has to deal with only a small number of queries, costs are typically low, and the policies should show good performance. Hence, the most interesting part of the parameter space is where $\rho_{1}$ is high and $\rho_{2}$ is low (we call this the critical region). We structure our numerical analysis accordingly, by first inspecting the performance of the policies for $0<\rho_{1} \leq 0.8,0<\rho_{2}<1$, followed by an inspection of the critical region $0.7<\rho_{1} \leq 1,0<\rho_{2}<0.2$. 
All numerical experiments below are done using the value iteration algorithm [20], and thus require a truncation of the state space $\mathcal{X}=\mathbb{N}_{0} \times \mathbb{N}_{0} \times \mathbb{N}_{0}$ to $\overline{\mathcal{X}}=\left[0, K_{1}\right] \times\left[0, K_{2}\right] \times\left[0, K_{3}\right]$. Choosing the $K_{i}$ must be done carefully to avoid the influence of boundary effects on the average costs. Tests on the three policies above, and on the optimal policy, show that a truncation to $\overline{\mathcal{X}}=[0,200] \times[0,200] \times[0,200]$ is sufficient for $0<\rho_{1} \leq 0.8,0<\rho_{2}<1$. Increasing $\rho_{1}$ beyond 0.8 quickly adds boundary effects and requires a larger truncated state space: $\overline{\mathcal{X}}=[0,300] \times[0,300] \times[0,300]$. Also, for value iteration we set the convergence criterion such that the procedure stops when the difference of the spans of two consecutive approximations is smaller than 0.001 .

Finally, we choose the parameters of the cost function in Eq. (1). We set $T=2, \gamma_{1}=\gamma_{2}=\gamma_{3}=3$ and keep these fixed during all experiments.

In the following sections we numerically investigate the performance of our improved policy. First, we compare the three policies listed above to the optimal policy in Sections 7.1 (for the non-critical region) and 7.2 (for the critical region). Then we look at the computational complexity in Section 7.3 by inspecting the time needed to calculate $\hat{\alpha}$ (and thus the improved policy), again compared to the time needed to find the optimal policy. Section 7.4 introduces a special random policy, where the controller flips a (fair) coin to decide which of the two actions to take. A large number of such policies are then compared to the three policies described above. Finally, in Section 7.5 we take a closer look at the optimal policy and its structure.

\subsection{Analysis of region $0<\rho_{1} \leq 0.8,0<\rho_{2}<1$}

In Figs. 5A $-5 \mathrm{C}$ we inspect the performance of the three policies as compared to the optimal policy. We fix $\mu_{1}=\mu_{2}=0.3$ and vary $\rho_{1}$ and $\rho_{2}$. The figures contain the difference in average costs with the optimal policy (in \%), where the load $\rho_{2}$ on $Q_{2}$ is varied on the horizontal axis, and the load $\rho_{1}$ of $Q_{1}$ is reflected by the various lines. Figs. 5A and $5 \mathrm{~B}$ show that the improved and myopic policies are able to stay within $1.3 \%$ and $5.5 \%$ of optimality, respectively. In contrast, the simple age-threshold policy differs from optimality by as much as $2,000 \%$. Clearly, the improved and myopic policies perform significantly better than the age-threshold policy, so including the load of the queue system in the decision by the controller certainly is beneficial. Further inspection of Figs. 5A and $5 \mathrm{~B}$ reveals that the performance of the three policies degrades when $\rho_{1}$ and $\rho_{2}$ reach the critical region. We take a detailed look at this region in the next section.

\subsection{Analysis of the critical region $0.7<\rho_{1} \leq 1,0<\rho_{2}<0.2$}

We continue with a closer look at the critical region, i.e., the left-hand side of Figs. 5A - 5C, by repeating the corresponding numerical experiments for different values of $\rho_{1}$ and $\rho_{2}$ (again with $\left.\mu_{1}=\mu_{2}=0.3\right)$. The results are in Figs. $6 \mathrm{~A}-6 \mathrm{C}$. As in the previous section, performance of the age-threshold policy is quite bad, with differences of up to 1,500\%. Comparing Figs. 6A to 6B clearly shows that the improved policy has better overall performance than the myopic policy, with differences from optimality of at most $7 \%$ and $17 \%$, respectively. The benefits of including the approximation to the value function in the improved policy are evident here.

Finally, Figs. 6A and 6B show that the relative differences are not monotone. The left-most points (at $\rho_{2}=0.01$ ) seem to be closer to optimality than the points at $\rho_{2}=0.05$. Further experiments suggest that this is not caused by boundary effects. Also, the differences cannot be explained by the stopping criterion of value iteration, because the differences are too large. Since the observed feature is present in both figures, it seems likely that the optimal policy causes it, and thus that this 


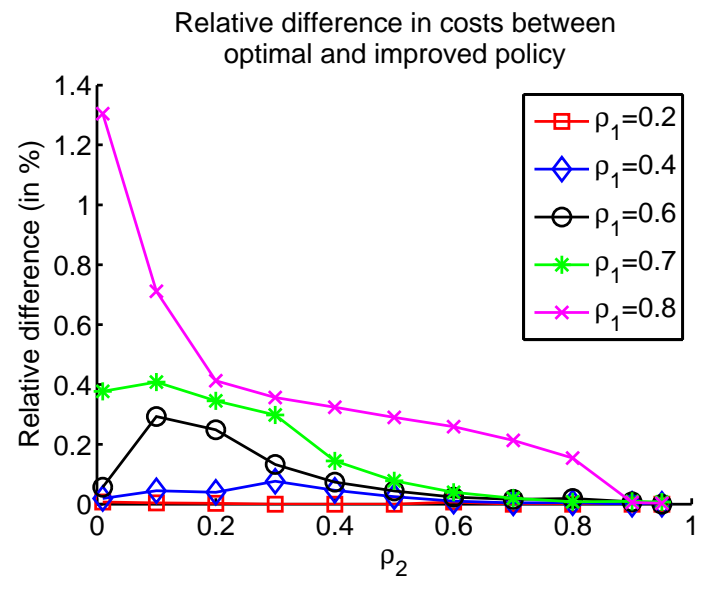

A

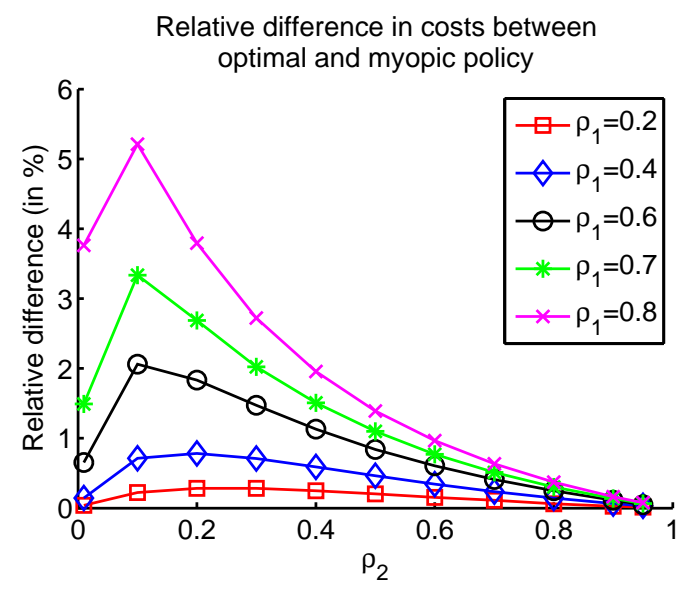

$\mathrm{B}$

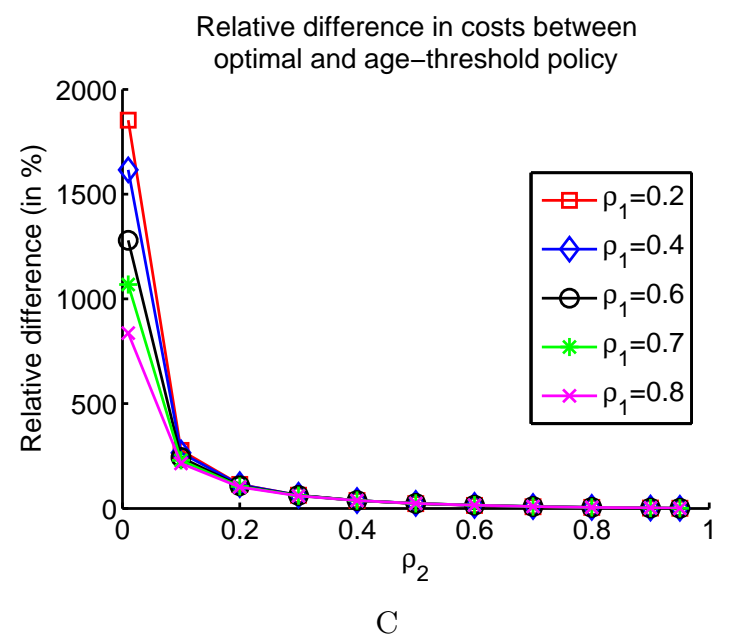

Figure 5. Relative difference in average costs of the improved policy (5A), myopic policy (5B), and agethreshold policy $(5 \mathrm{C})$ compared to the optimal policy

behavior is a feature of the system. We return to this topic later in Section 7.5 when we talk about the optimal policy.

\subsection{Computational complexity}

As described in Section 6.2, the improved policy requires four short runs of the value iteration algorithm to determine the parameter $\hat{\alpha}$. The total duration of these runs should be less than the time required to find the optimal policy. Table 1 shows the time needed to find $\hat{\alpha}$ for the improved policy, divided by the time required to determine the optimal policy. As parameter values we use the same scenario as in Section 7.2, i.e., $\mu_{1}=\mu_{2}=0.3$. The two tables clearly show that determining the improved policy is much faster than finding the optimal policy.

\subsection{Model complexity}

To get a feel for the complexity of the model in Eq. (2), we plot a so-called Ordered Performance Curve (OPC) [10]. Each point in this plot shows the average costs of a policy that we generate 


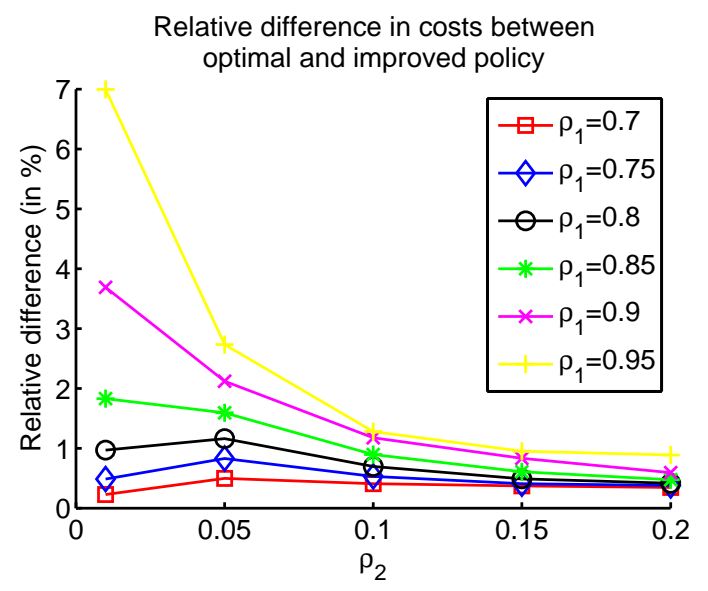

A

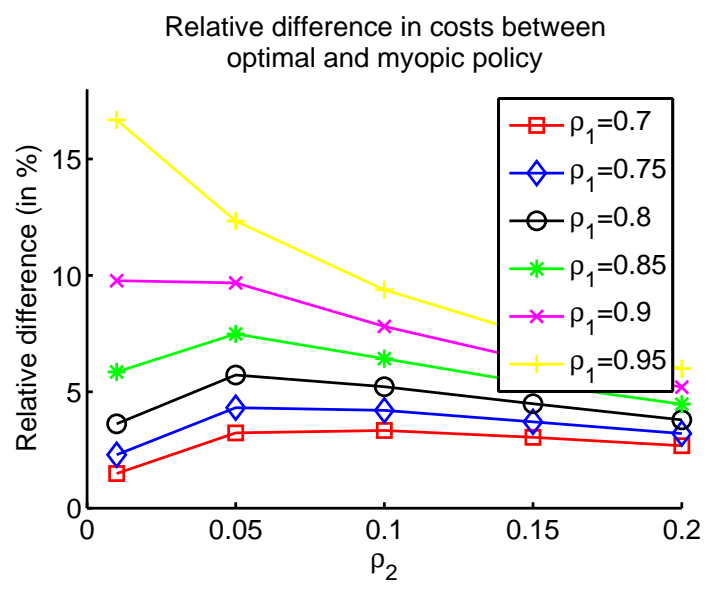

$\mathrm{B}$

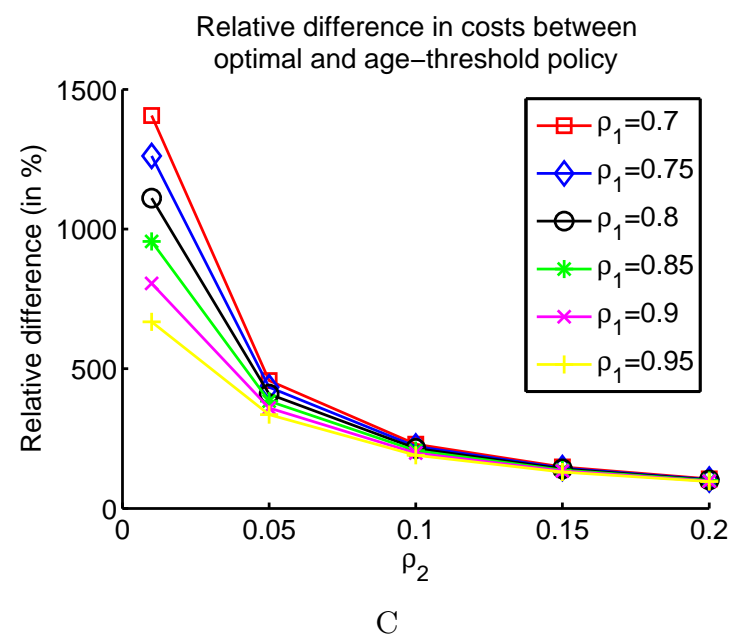

Figure 6. Again, the relative difference in average costs of the improved policy (6A), myopic policy (6B), and age-threshold policy (6C) compared to the optimal policy, but now inside the critical region

randomly: at each state $(i, j, N)$ we choose action $a=\left\{Q_{1}\right\}$ with probability 0.5 , or $a=\{D B\}$ otherwise. By repeating this procedure, we create 2,500 such policies, evaluate them, and plot their average costs in Fig. 7A. Additionally, this figure shows the average costs of the optimal policy and (in our case) of the improved, the age-threshold, and myopic policies. The parameters are $\mu_{1}=\mu_{2}=0.3, \rho_{1}=0.8, \rho_{2}=0.1$, based on the critical region in the parameter space. Since the markers of the optimal, improved, and myopic policies are indistinguishable in Fig. 7A, the 15 best policies are plotted again in Fig. 7B. The steep slope on the left of both figures illustrates that none of the randomly selected policies is able to closely match the performance of the optimal policy. Hence, the plot suggests that the near-optimal performance of the improved policy shown in Figs. $5 \mathrm{~A}-6 \mathrm{C}$ is not an incidental success. Moreover, one can also see that the traditional age-threshold policy performs badly, showing a lot of room for improvement by using dynamic policies.

\subsection{The optimal policy}

Next, we inspect the optimal policy in Figs. $8 \mathrm{~A}$ and $8 \mathrm{~B}$. The first shows a cross-section of the optimal policy at $N=55$, the second at $N=120$. Here, for every grid point $(i, j)$ the color gray indicates that action $a=\mathrm{DB}$ is taken and black that $a=Q_{1}$. The figures suggest that (away from the boundaries) the optimal policy is a hyperplane in three-dimensional space, i.e., a switching policy. 


\begin{tabular}{cc|cccccc} 
& & \multicolumn{6}{|c}{$\rho_{1}$} \\
& & 0.7 & 0.75 & 0.8 & 0.85 & 0.9 & 0.95 \\
\hline \multirow{4}{*}{$\rho_{2}$} & 0.01 & 0.0122 & 0.0061 & 0.0065 & 0.0059 & 0.0054 & 0.0054 \\
& 0.05 & 0.0048 & 0.0069 & 0.0068 & 0.0076 & 0.0070 & 0.0069 \\
& 0.10 & 0.0060 & 0.0058 & 0.0070 & 0.0067 & 0.0078 & 0.0077 \\
& 0.15 & 0.0056 & 0.0054 & 0.0067 & 0.0063 & 0.0075 & 0.0061 \\
& 0.20 & 0.0064 & 0.0063 & 0.0061 & 0.0071 & 0.0070 & 0.0068
\end{tabular}

Table 1. The run time for determining $\hat{\alpha}$ divided by the run time needed to obtain the optimal policy for various parameter settings

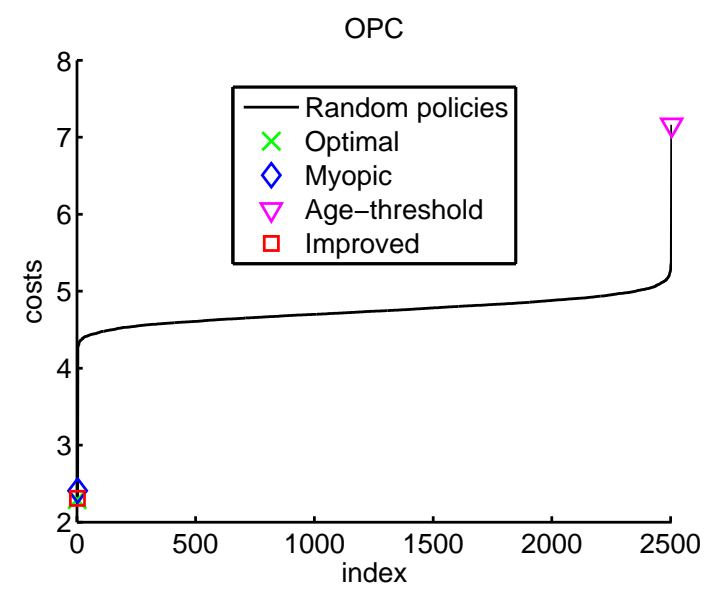

A

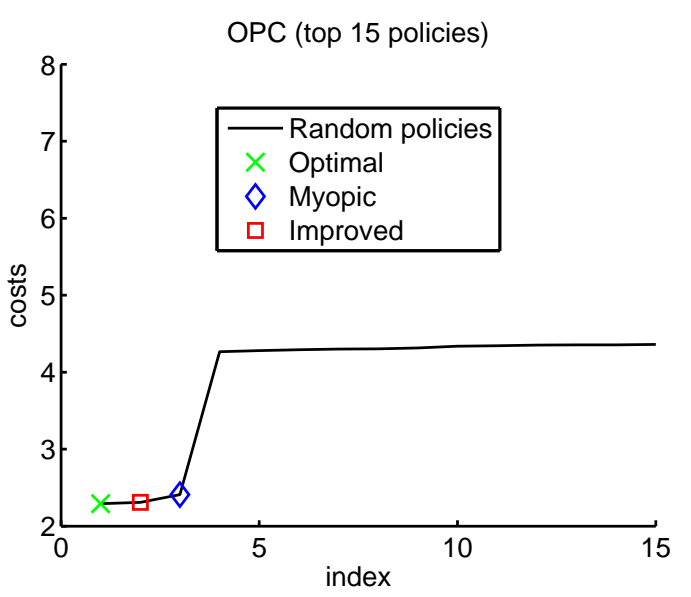

$\mathrm{B}$

Figure 7. Ordered Performance Curve - costs of 2,500 randomly selected policies, as well as the optimal, improved, and myopic policies. The markers of the latter three policies are difficult to distinguish in Fig. 7A, so Fig. 7B shows only the 15 best policies

This observation is supported by intuitions about the problem scenario: once $Q_{1}$ reaches a certain load, the controller switches to using the DB, and continue to do so as the load increases. Hence, an optimal policy with a switching structure is in line with our expectations. Hence, for $N>T$ we expect by intuition a switching structure in the optimal policy, which is confirmed by what we see in Figs. 8A and 8B. We were unable to verify this last observation mathematically, but we expect that a proof is feasible. The conjecture below formalizes the claim:

Conjecture 1 (Asymptotic switching policy). The optimal policy for the MDP in Eq. (2) is a switching curve for $N$ sufficiently large.

Looking at Figs. 8A and 8B, we see that the optimal policy is cropped near the boundary $\{j=0\}$. This effect is caused by the interaction between the number of report requests $j$ and the costs $(N-T)^{+}$for DB assignments. They are connected via $N$ using the term $\mu_{2} V(i, j-1,0) \mathbb{1}_{\{j>0\}}$ in Eq. (2), which drops out at the boundary $\{j=0\}$. Consequently, on the boundary the connection between $j$ and $N$ is severed, and changes the structure of the MDP and the optimal policy significantly. This also explains the observation in Section 7.2 that the performance of the improved and myopic policies changes for $\rho_{2} \approx 0$.

Still, in situations where the boundary $\{j=0\}$ is not reached frequently, we expect switching policies to perform well since the boundary effect is relatively small. This is supported by the results on our improved policy and the myopic policy (both are switching policies) in the previous sections. 


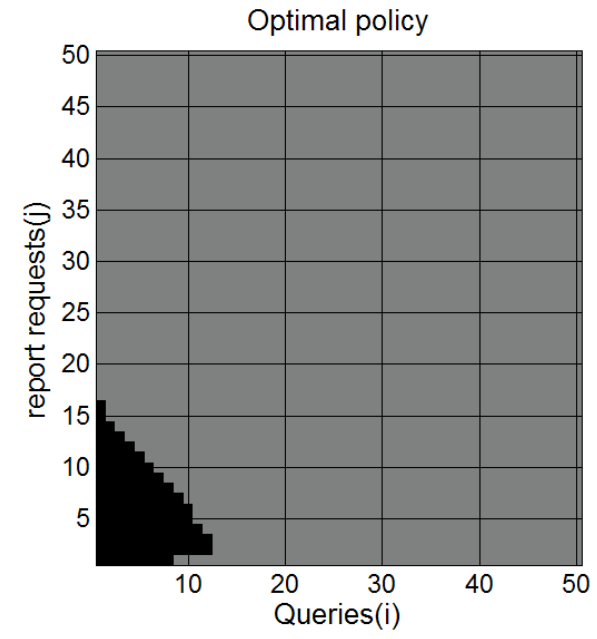

A $N=55$

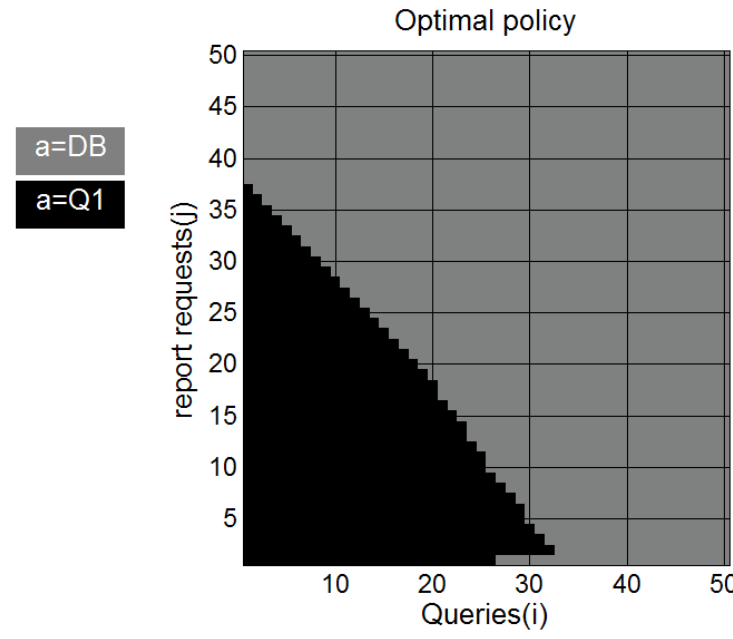

B $N=120$

Figure 8. Optimal policy for $N=55(8 \mathrm{~A})$ and $N=120(8 \mathrm{~B})$. Gray indicates that action $a=$ DB is taken, black $a=Q_{1}$. In these figures, we again use parameters $\mu_{1}=\mu_{2}=0.3, \rho_{1}=0.8, \rho_{2}=0.1$ from the critical region

\section{Conclusions and further research}

In this paper we investigated the trade-off between data freshness and query response times. We formulated this trade-off as a Markov Decision Process with a three-dimensional state space. The resulting model contained two complicating aspects: (1) a decision capturing the trade-off, with several inhomogeneous terms and (2) intricate interactions between state space variables. Due to these complications, obtaining an analytical expression for the optimal policy was infeasible. Instead, we introduced a three-step approach to finding an approximate policy with near-optimal performance. The first step showed how the original three-dimensional model can be approximated by a simpler two-dimensional model that still captures the important dynamics. Then, in the second step, we described how this simpler model can be solved analytically, using differencing techniques to deal with the inhomogeneous terms. In step three we applied one-step policy improvement to construct our approximate policy. Finally, we numerically showed that this improved policy has near-optimal performance, and significantly outperforms both the traditional age-threshold policy and the myopic policy. The experiments also indicated that the policies that take the network load into account (the myopic and improved policy) can outperform traditional threshold policies.

The research in this paper reveals several interesting opportunities for further research. We would like to modify our improved policy such that it no longer requires short runs of value iteration for determining the parameter $\alpha$. Also, we suspect that we can prove some structural properties of the optimal policy using coupling arguments: (1) queries are more likely to go to DB if i increases, holding $j$ and $N$ constant, (2) queries are more likely to go to $Q_{1}$ if $N$ increases, holding $i$ and $j$ constant, and (3) for $j$ large enough, queries will go to DB, holding $i$ and $N$ constant. If these structural properties are shown to hold, they would imply our conjecture that the optimal policy is asymptotically a switching curve. Finally, we want to take a closer look at the critical region of the state space and attempt to mathematically analyze what happens when the load on the report queue approaches 0 .

Besides these ideas, a modification of the model where the cost of function is formulated differently (e.g., not truncated at the threshold $T$ or non-linear) might be interesting. Additionally, the model could be extended by considering multi-class queries, where the cost function is dependent on the class of the arriving query. 


\section{Acknowledgements}

This work was performed within the project RRR (Realisation of Reliable and Secure Residential Sensor Platforms) of the Dutch program IOP Generieke Communicatie, number IGC1020, supported by the Subsidieregeling Sterktes in Innovatie. We thank SURFsara [2] for the support in using the Lisa Compute Cluster.

\section{References}

[1] Apache webserver documentation - http://httpd.apache.org/docs/2.4/caching.html.

[2] SURFSara - http://www.surfsara.nl.

[3] E. Altman, B. Gaujal, A. Hordijk, and G. Koole. Optimal admission, routing and service assignment control: the case of single buffer queues. In Decision and Control, 1998. Proceedings of the 37th IEEE Conference on, volume 2, pages 2119-2124. IEEE, 1998.

[4] S. Bhulai. Markov decision processes: the control of high-dimensional systems. PhD thesis, Universal Press, Netherlands, 2002.

[5] S. Bhulai. Dynamic routing policies for multiskill call centers. Probability in the Engineering and Informational Sciences, 23(1):101, 2009.

[6] S. Bhulai and G. Koole. On the structure of value functions for threshold policies in queueing models. Journal of Applied Probability, pages 613-622, 2003.

[7] M. Dahlin. Interpreting stale load information. Parallel and Distributed Systems, IEEE Transactions on, 11(10):1033-1047, 2000.

[8] R. Haijema and J. Van der Wal. An MDP decomposition approach for traffic control at isolated signalized intersections. Probability in the Engineering and Informational Sciences, 22(04):587602,2008 .

[9] O. Hernández-Lerma and J. B. Lasserre. Discrete-Time Markov Control Processes: Basic Optimality Criteria. Springer Verlag, 1996.

[10] Y. Ho, Q. Zhao, and Q. Jia. Ordinal Optimization: Soft Optimization for Hard Problems. Springer, September 2007.

[11] S. A. Lippman. Applying a new device in the optimization of exponential queuing systems. Operations Research, 23(4):687-710, July 1975.

[12] Mihaela Mitici, Martijn Onderwater, Maurits de Graaf, Jan-Kees van Ommeren, Nico van Dijk, Jasper Goseling, and Richard J. Boucherie. Optimal query assignment for wireless sensor networks. International Journal of Electronics and Communications (AEU), 69(8):1102 - 1112, 2015.

[13] M. Mitzenmacher. How useful is old information? Parallel and Distributed Systems, IEEE Transactions on, 11(1):6-20, 2000.

[14] J. M. Norman. Heuristic procedures in dynamic programming. Manchester University Press, 1972.

[15] T. J. Ott and K. R. Krishnan. Separable routing: A scheme for state-dependent routing of circuit switched telephone traffic. Annals of operations research, 35(1):43-68, 1992. 
[16] RFC5861. http://www.rfc-editor.org/rfc/rfc5861.txt.

[17] S. A. E. Sassen, H. C. Tijms, and R. D. Nobel. A heuristic rule for routing customers to parallel servers. Statistica Neerlandica, 51(1):107-121, 1997.

[18] R. F. Serfozo. An equivalence between continuous and discrete time Markov decision processes. Operations Research, 27(3):616-620, May 1979.

[19] F. M. Spieksma. Geometrically ergodic Markov chains and the optimal control of queues. PhD thesis, Rijksuniversiteit Leiden, Leiden, 1990.

[20] D.J. White. Dynamic programming, Markov chains, and the method of successive approximations. Journal of Mathematical Analysis and Applications, 6:373-376, 1963.

[21] J. Wijngaard. Decomposition for dynamic programming in production and inventory control. Engineering and process economics, 4(2):385-388, 1979.

\section{A Uniqueness of solutions}

In Section 5 we solved the two-dimensional difference equation (5), known in MDP literature as the Poisson equation. For this equation we have only one boundary condition $V(0,0)=0$, which is not enough to completely determine the solution. Consequently, after solving the difference equation the constant $\tilde{V}_{1}^{\alpha}(0)$ is still to be determined in Eq. (20).

In order to investigate uniqueness we repeat arguments from Chapter 2 and 4 of [4]. First, note that Eq. (5) induces a Markov cost chain with transition matrix $P$, state space $\mathcal{X}=\mathbb{N}_{0} \times \mathbb{N}_{0}$, and cost function $c(i, j)=\lambda_{1} \alpha\left[\gamma_{1}(i+1)+\gamma_{2} j+\gamma_{3}\right]+\lambda_{1}(1-\alpha) C$. Denote with $\mathbb{B}(\mathcal{X})$ the Banach space of bounded real-valued functions $u$ on $\mathcal{X}$ with the supremum norm, i.e., the norm $\|\cdot\|$ defined by

$$
\|u\|=\sup _{(i, j) \in \mathcal{X}}|u(i, j)| .
$$

Conventional uniqueness proofs for Markov cost chains rely on bounded cost functions contained in $\mathbb{B}(\mathcal{X})$. However, our cost function $c(i, j)$ is unbounded and thus not contained in $\mathbb{B}(\mathcal{X})$. A remedy to this situation is to consider suitable larger Banach spaces instead of $\mathbb{B}(\mathcal{X})$. In order to construct such a space, consider a weight function $w: \mathcal{X} \rightarrow[1, \infty)$. The $w$-norm is then defined by

$$
\|u\|_{w}=\sup _{(i, j) \in \mathcal{X}} \frac{|u(i, j)|}{w(i, j)}
$$

A function $u$ is said to be $w$-bounded if $\|u\|_{w}<\infty$, and the space of all $w$-bounded functions is denoted by $\mathbb{B}_{w}(\mathcal{X})$. We also define the matrix norm related to $\|\cdot\|_{w}$ as $\|A\|_{w}=\sup \left\{\|A u\|_{w}:\|u\|_{w} \leq 1\right\}$. This norm can be rewritten in the following equivalent form (see Eq. (7.2.8) in [9])

$$
\|A\|_{w}=\sup _{x \in \mathcal{X}} \sum_{y \in \mathcal{X}} \frac{\left|A_{x y}\right| w(y)}{w(x)} .
$$

Finally, we introduce the taboo transition matrix ${ }_{M} P$ as

$$
{ }_{M} P_{x y}=\left\{\begin{aligned}
P_{x y}, & y \neq M, \\
0, & y \in M,
\end{aligned}\right.
$$

with $x, y \in \mathcal{X}$ and in our case $M=(0,0)$. We now state a property and adapted theorem from [4] on uniqueness of solutions of Eq. (5). 
Property 1 (page 19 of [4]). A Markov chain is called w-geometrically recurrent with respect to $M$ $[w-G R(M)]$ if there exists an $\epsilon>0$ such that $\left\|{ }_{M} P\right\|_{w} \leq 1-\epsilon$.

Theorem 2 (Lemma 2.1 combined with Theorem 2.10 of [4]). Suppose that the Markov chain induced by a policy $\pi$ is unichain, stable, aperiodic, and $w$-GR(M). Let both $(g, V)$ and $\left(g^{\prime}, V^{\prime}\right)$ be solutions to the Poisson equation. Then $g=g^{\prime}$ and the value functions $V$ and $V^{\prime}$ differ by only a constant.

In our case, the Bernoulli policy does indeed induce a Markov chain that is unichain, stable, and aperiodic. The key to ensuring uniqueness is choosing a suitable weight function $w$ such that Property 1 is satisfied. Section 3.4 of [19] shows that a suitable weight function is of the form

$$
w(i, j)=K \prod_{k=1}^{i}\left(1+m_{k}\right) \prod_{l=1}^{j}\left(1+n_{l}\right),
$$

where $\left\{m_{k}\right\},\left\{m_{l}\right\}$, and $K$ are constants. Unfortunately, the expressions involved are cumbersome and not easy to state explicitly, making it difficult for us to illustrate the construction of the weight function. In the remainder of this section we make an additional assumption that allows us to find a weight function that is explicit. This assumption is only made to facilitate explicitness, and readers interested in the case without the assumption are referred to [19].

Following Section 4.1 of [4], we assume that $\rho_{1} \alpha+\rho_{2}<1$. The non-zero entries in transition matrix are given by

$$
\begin{aligned}
& P_{(i, j)(i+1, j)}=\lambda_{1} \alpha \\
& P_{(i, j)(i, j+1)}=\lambda_{2} \\
& P_{(i, j)(i-1, j)}=\mu_{1} \mathbb{1}_{\{i>0\}}, \\
& P_{(i, j)(i, j-1)}=\mu_{2} \mathbb{1}_{\{j>0\}}, \\
& P_{(i, j)(i, j)}=1-P_{(i, j)(i+1, j)}-P_{(i, j)(i, j+1)}-P_{(i, j)(i-1, j)}-P_{(i, j)(i, j-1)} .
\end{aligned}
$$

Set $w(i, j)=\left(1+k_{1}\right)^{i}\left(1+k_{2}\right)^{j}$ for some constants $k_{1}$ and $k_{2}$. Now consider

$$
\left\|_{M} P\right\|_{w}=\sum_{\left(i^{\prime}, j^{\prime}\right) \neq(0,0)} \frac{P_{(i, j)\left(i^{\prime}, j^{\prime}\right)} w\left(i^{\prime}, j^{\prime}\right)}{w(i, j)}
$$

which is given by

$$
\begin{cases}\lambda_{1} \alpha\left(1+k_{1}\right)+\lambda_{2}\left(1+k_{2}\right), & (i, j)=(0,0), \\ \lambda_{1} \alpha k_{1}+\lambda_{2} k_{2}+1-\mu_{1}, & (i, j)=(1,0), \\ \lambda_{1} \alpha k_{1}+\lambda_{2} k_{2}+1-\mu_{2}, & (i, j)=(0,1), \\ \lambda_{1} \alpha k_{1}+\lambda_{2} k_{2}+1-\frac{\mu_{1} k_{1}}{1+k_{1}}, & i>1, j=0, \\ \lambda_{1} \alpha k_{1}+\lambda_{2} k_{2}+1-\frac{\mu_{2} k_{2}}{1+k_{2}}, & i=0, j>1, \\ \lambda_{1} \alpha k_{1}+\lambda_{2} k_{2}+1-\frac{\mu_{1} k_{1}}{1+k_{1}}-\frac{\mu_{2} k_{2}}{1+k_{2}}, & i>0, j>0 .\end{cases}
$$

We need to choose $k_{1}$ and $k_{2}$ such that all expressions are strictly less than 1 . Observe that if the fourth and fifth expression are less than 1 , then all others are also satisfied. Hence, we can restrict 
our attention to the system

$$
\begin{aligned}
& f_{1}\left(k_{1}, k_{2}\right)=1+\lambda_{1} \alpha k_{1}+\lambda_{2} k_{2}-\frac{\mu_{1} k_{1}}{1+k_{1}}, \\
& f_{2}\left(k_{1}, k_{2}\right)=1+\lambda_{1} \alpha k_{1}+\lambda_{2} k_{2}-\frac{\mu_{2} k_{2}}{1+k_{2}},
\end{aligned}
$$

with the assumptions $\lambda_{1} \alpha+\lambda_{2}+\mu_{1}+\mu_{2}<1$ and $\rho_{1}+\rho_{2}<1$.

Observe that $f_{1}(0,0)=f_{1}\left(\left(\mu_{1}-\lambda_{1} \alpha\right) /\left(\lambda_{1} \alpha\right), 0\right)=1$. Thus, the points $(0,0)$ and $\left(\left(\mu_{1}-\lambda_{1} \alpha\right) /\left(\lambda_{1} \alpha\right), 0\right)$ lie on the curve $f_{1}\left(k_{1}, k_{2}\right)=1$. Furthermore, $k_{2}$ satisfies $k_{2}=\mu_{1} / \lambda_{2}-\mu_{1} /\left(\lambda_{2}\left(1+k_{1}\right)\right)-\lambda_{1} \alpha / \lambda_{2}$. Note that this function has a maximum value at $k_{1}=\sqrt{\mu_{1} /\left(\lambda_{1} \alpha\right)}-1$. Hence, this description determines the form of $f_{1}$; the curve $f_{1}\left(k_{1}, k_{2}\right)=1$ starts in $(0,0)$ and increases to an extreme point, and then decreases to the $k_{1}$-axis again. The curve $f_{2}$ has a similar form, but with the role of the $k_{1}$-axis interchanged with the $k_{2}$-axis.

The curves determine an area of points $\left(k_{1}, k_{2}\right)$ such that $f_{1}$ and $f_{2}$ are strictly less than one if the partial derivative to $k_{1}$ at $(0,0)$ of the curve $f_{1}\left(k_{1}, k_{2}\right)=1$ is greater than the partial derivative to $k_{2}$ of the curve $f_{2}\left(k_{1}, k_{2}\right)=1$ at $(0,0)$. These partial derivatives are given by $\left(\mu_{1}-\lambda_{1} \alpha\right) / \lambda_{2}$ and $\lambda_{1} \alpha /\left(\mu_{2}-\lambda_{2}\right)$, respectively. Since $\rho_{1} \alpha+\rho_{2}<1$, we have $\lambda_{1} \alpha \mu_{2}+\lambda_{2} \mu_{1}<\mu_{1} \mu_{2}$. Adding $\lambda_{1} \alpha \lambda_{2}$ to both sides gives $\lambda_{1} \alpha \lambda_{2}<\mu_{1} \mu_{2}-\lambda_{1} \alpha \mu_{2}-\lambda_{2} \mu_{1}+\lambda_{1} \alpha \lambda_{2}=\left(\mu_{1}-\lambda_{1} \alpha\right)\left(\mu_{2}-\lambda_{2}\right)$. Hence, the relation $\lambda_{1} \alpha /\left(\mu_{2}-\lambda_{2}\right)<\left(\mu_{1}-\lambda_{1} \alpha\right) / \lambda_{2}$ holds. Thus, indeed the partial derivative to $k_{1}$ at $(0,0)$ of the curve $f_{1}\left(k_{1}, k_{2}\right)=1$ is greater than the partial derivative to $k_{2}$ of the curve $f_{2}\left(k_{1}, k_{2}\right)=1$ at $(0,0)$, and there is an area of pairs $\left(k_{1}, k_{2}\right)$ such that the Markov chain is $w$-GR(M). For these points it holds that $\left(1+k_{n}\right)<1 / \rho_{n}$ for $n=1,2$. Observe that any sphere with radius $\epsilon>0$ around $(0,0)$ has a non-empty intersection with this area. Hence, the cost function cannot contain terms in $i$ and/or $j$ that grow exponentially fast to infinity, and neither can the value function. Consequently, we need to choose $\tilde{V}_{1}^{\alpha}(0)$ in Eq. (20) such that the exponential term $\left(\frac{\mu_{1}}{\lambda_{1} \alpha}\right)^{i}$ disappears. 